\title{
Self-Assembly of Organic Supramolecular Capsules
}

\author{
Alessandro Scarso and Giuseppe Borsato \\ Università Ca' Foscari di Venezia, Venezia, Italy
}

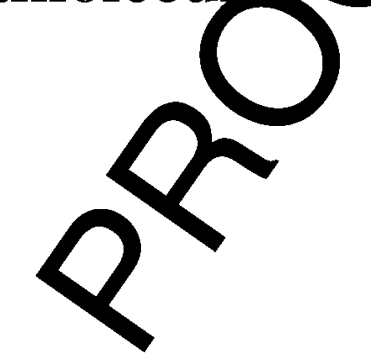

1 Introduction

2 Hydrogen-bonding Homomeric Capsules

3 Hydrogen-bonding Heteromeric Capsules

4 Ion-pairing Heteromeric Capsules

5 Hydrophobic Capsules

6 Conclusions

Acknowledgments

Further Reading

References

\section{INTRODUCTION}

\subsection{Supramolecular capsules}

Supramolecular self-assembled organto apsules are formed when more than one unit, charctens d by the same or different chemical nature, spont feously $y$ counter in solution giving rise to an aggregate tha forms cavity where guest molecules can be acco modated. Driving forces for this "get together" that invo ves sevg fal partners can be driven by either the enthal an when working in apolar media, or the entropic an antage when the aggregate forms in polar solvents turagende behaves as a new chemical entity, and it ten not have much to share with the components. Usua, the subunits that compose the capsule featuro haveave hapes and are adorned with functional groups that in agregate are involved in weak intermolecular interactions like hydrogen bonding, ion pairing,

Supramolecular Chemistry: From Molecules to Nanomaterials. Edited by Philip A. Gale and Jonathan W. Steed.

(c) 2011 John Wiley \& Sons, Ltd. ISBN: 978-0-470-74640-0. or hydr interactions. For efficient aggregation, the prese fe of $\mathrm{ml}$ ivalent functional groups in every subunit is requo a cooperativity between attractive interacti ns is often observed. ${ }^{1}$ Usually, subunits present a certain ar of gidity and a limited number of possible conforman ecause this strongly favors intermolecular interactions and reciprocal docking, but this observation should no be taken as a rule because in many cases it is the bound guest within the cavity that drives the formation of a certain aggregate imparting preferential conformations to the subunits that can be adopted if they are semiflexible. Overall, the formula for assembly is written in the specific kind of functional groups, in molecular curvature present in the subunits, together with adequate filling of the cavity.

An important feature of the self-assembled capsule is the reversibility of the aggregation process that can be inverted by correct changes in the experimental conditions. Capsules are not immobile systems, rather there is a continuous dynamic exchange of subunits at rates that are a function of the number and strength of intermolecular interactions. It is because of these phenomena that the cavity present in capsules can be accessed by external guests. Common methods of monitoring and characterizing guest exchange are several, like NMR (nuclear magnetic resonance), UV-vis, mass spectrometry, electrochemistry, and calorimetry. ${ }^{2}$

The same concepts are present in nature that has evolved examples of natural supramolecular capsules deputed to the transport and/or the storage of important chemical species. Examples are the tobacco mosaic virus characterized by a rodlike appearance and composed of 2130 molecules of coat protein that surround one molecule of genomic RNA 6400 bases long. Another important representative is apoferritin, which is a capsular aggregate with octahedral symmetry formed by 24 identical peptides that present a cavity of over $230 \AA^{3}$ that can store up to 4500 iron atoms as ferric hydrous oxides. 


\subsection{Lifetime of capsules}

As far as aggregation phenomena in solution are concerned, it is worth noting that when two molecules come together and no special attractive interactions are present among them, the lifetime of this encounter complex is on the order of $<10^{-9} \mathrm{~s}$. This means that if we follow the fate of one single molecule for $1 \mathrm{~s}$, this embarks in intermolecular contacts with several millions of solvent molecules in an extremely rapid change of partners. If we connect several of these solvent molecules and arrange them in a concave shape, the result is longer contacts between the molecule and the aggregates and reduced number of possible partners. If we extend this concept to a much smaller number of species, we observe the same phenomena that occur to guests in self-assembled capsules. The capsule acts as an organized solvent where different residues have been brought together and the entropic penalty for their organization has already been paid through the synthesis of the hosting subunits. This translates into much longer lifetimes for capsule-guest interactions that can last up to seconds. In other words, while in the bulk solvent single molecule senses a huge number of other molecu for extremely short times, in capsules the guest interacts with the same capsule from $10^{3}$ to $10^{-3} \mathrm{~s}$, having ough time to be strongly reciprocally influenced..$^{3}$

Ingress and egress of the guest from the via different supramolecular mechanisms from the temporary disassembly of one sut a door through which the guest goes alternatively, opening flaps of subunit dissociation, thus limiting the enthar ost. In detail, substitution of guests in capsules y ly do via either a bimolecular guest substitution hen ancoming guest appears in the rate expression ( $S_{2}$ or via unimolecular

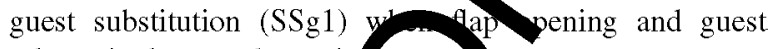
release is the rate-determin ig step.

In order to follow capsu format on and guest binding, it is necessary to use to that able to "take snapshots" of the system at a rat faster 4 mpared to the process under investigation, thus alld ving th detection of signals for each species present in sytion. If the technique employed is not fast enoug average of the exchanging systems is obseryed cause of the exchange that occurs during the time requn forobservation of the system, with signals in-ween th species that exchanged during the shot. NMR specoscos is the most adequate/prevalent technique because, with the opportune tuning of the temperature, it is possible to regulate the exchange process between fast and slow exchange regime that, compared to the chemical shift timescale, falls in the range from $0.1 \mathrm{~ms}$ up to a few seconds. IR and UV-vis spectroscopy are instead in the range of picoseconds; therefore, the ste on served almost always reside in the slow exchy ge regime

Moreover, the encapsulation proce can easnly studied because NMR resonances of the trap guests are shifted upfield by the aromatic ring crost, often kinetically stable, and in sld excha ge on the NMR chemical shift timescale, revea ng two istinct species for the encapsulated and free

\subsection{The viewpoimot grest}

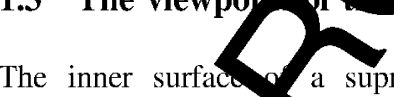

The inner surfaco a supramolecular capsule is not different fron sure of any other molecule; both require con cts $y$ th other molecular species in order to minimize the erall energy of the system as much as possible; basically, internal surfaces need to be solvated as my other molecules in solution. Because of the clo d struct e and the prolonged contact times, the bound polect is hore strictly selected compared to solvation henmento in the bulk and extra requisites are necessary to tro sfory simple molecule into a suitable guest. Matching or ha $\mathrm{C}$ is obviously required and, as far as the size is concerned, again a parallel with what happens in solvents natural. It has been observed that bulk solvents are characterized by packing coefficient (PC) values, that is, the volume of all the molecules that occupy a certain volume of a liquid, which is in the range of $45-55 \%$. A close observation of several capsule-guest interactions demonstrated that the same criteria apply to encapsulation phenomena. ${ }^{6}$ In fact, good binding can be expected if the $\mathrm{PC}$ is in the range $0.55 \pm 0.09$ for liquid guests, while for gaseous guests the value is smaller, around 0.4 , implying that the gas in the capsule behaves similar to gas molecules in the gas phase even in condensed matter.

Apart from the above-mentioned criteria, the guest interacts with the host, establishing attractive or repulsive weak intermolecular forces with the capsule following criteria that are well known in supramolecular chemistry like hydrogen bonding, $\pi-\pi$ stacking, $\mathrm{CH}-\pi$, cation and anion $-\pi$ interactions, and so on. For large capsules that can accommodate more than a single guest, interguest contacts are possible with both being isolated from the rest of the solvent with the opportunity to observe molecule-to-molecule interactions. ${ }^{7}$ Encapsulation of guests ${ }^{8}$ allows new phenomena such as supramolecular stereochemistry ${ }^{9-11}$ restricted tumbling, and spinning and modified conformations to emerge, ${ }^{12}$ most of which are described in the following.

The ultimate target of self-assembly of capsules and guest binding is the mimicry of what happens in nature. In particular, two phenomena are the source of inspiration: the transport of molecules where the capsule acts as a reversible carrier that takes up the guest in a place and releases it in 
another upon opportune stimulus, and the catalysis where the capsule acts as an enzyme that binds substrates forming a complex that evolves to products and free catalyst ready for a subsequent cycle. Review articles covering various aspects of these phenomena are reported in the section titled "Further Reading".

Significant examples of organic self-assembled capsules that have been fully studied in solution along with a discussion on their peculiar encapsulation behaviors, catalogued on the basis of the kind of intermolecular force involved like hydrogen bonding, ion pairing, and hydrophobic effect in increasing order of number and kind of units that compose the supermolecule are illustrated in the following. The focus is on the interactions that emerge within the cavity between the guests bound and the self-assembled capsule, citing examples of capsules that do not show clear examples of encapsulation only if they represent in any way an advancement in the field.

\section{HYDROGEN-BONDING HOMOMERIC CAPSULES}

In the present section, capsules held together by hydroge bond interactions in noncompetitive organic solvents increasing size and complexity order from dimep to multimeric aggregates are described. Capsules ma e of all equal units (homomeric capsules) are illustry followed by those comprising different units capsules).
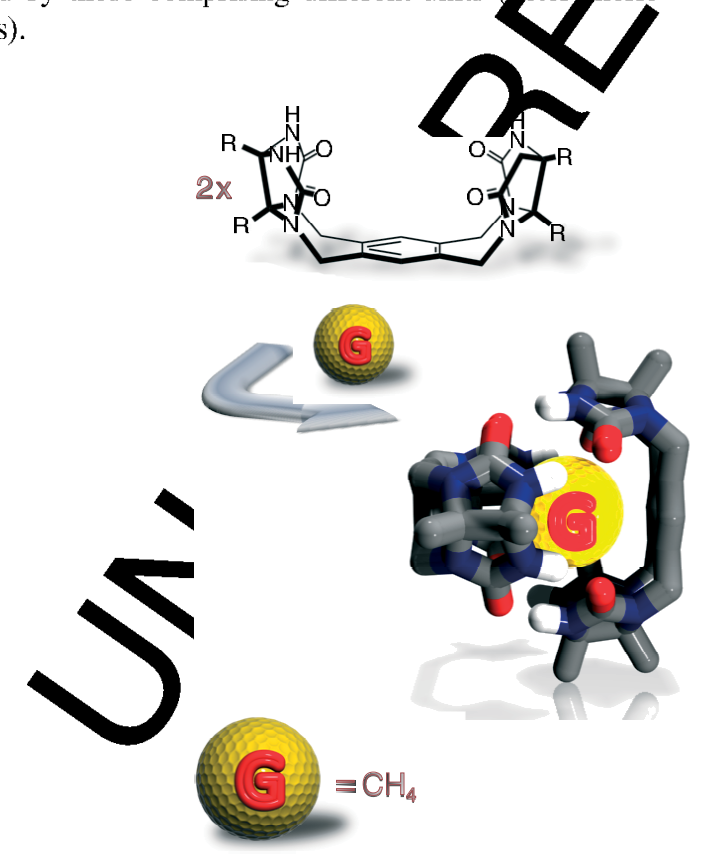

\subsection{Dimeric capsules}

\subsubsection{Tennis ball}

The first hydrogen-bonded supramolecula sapsule reported was developed by Rebek and coly work and was based on the connection between two o ycoluril nits to a durene scaffold, giving rise to a U-shap mole ule featuring an array of hydrogen-bonding csan on periphery that provide overall eight hydrog ( bonds seal the capsule and form a cavity of approxim ely $55 \Omega^{3,13}$ In chloroform- $d$, the system shows tw sp cies in agreement with an open and a dimeric clos strutu in slow exchange on the NMR timescale (Figh 1). The cavity is rather small and can accommoden fuid gusts like dichloromethane, but more singula the capsule showed clear encapsulation of gaseous guests ${ }^{\mathrm{k}} \mathrm{e}$ methane, ethane, and ethylene with decreasing hinding constants. A smaller capsule, where the aromat rest was present on the scaffold, derived by duren was re aced by a tetrasubstituted double bond ${ }^{14}$ ob Ining -assembled host system that did not encaprate chloromethane and that showed higher selectivity to as b Ading with much higher affinity for methane compo to that for ethane. Substitution of the aromatic aacer of the monomer with electron-rich hydroquinone allored encapsulation of fluorinated gusts like $\mathrm{CH}_{3} \mathrm{~F}$ and $\mathrm{CF}_{4}$ investigated by ${ }^{19} \mathrm{~F}$ NMR where separate signals for free and bound guest showed higher affinity for the former. ${ }^{15}$ Other permutations on this kind of capsule led to the formation of a chiral tennis ball starting from an achiral monomer where two different glycoluril residues were

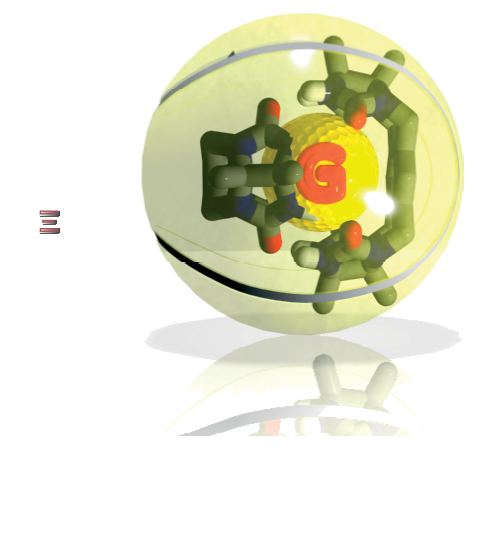

Figure 1 Homodimeric tennis ball encapsulating methane. 


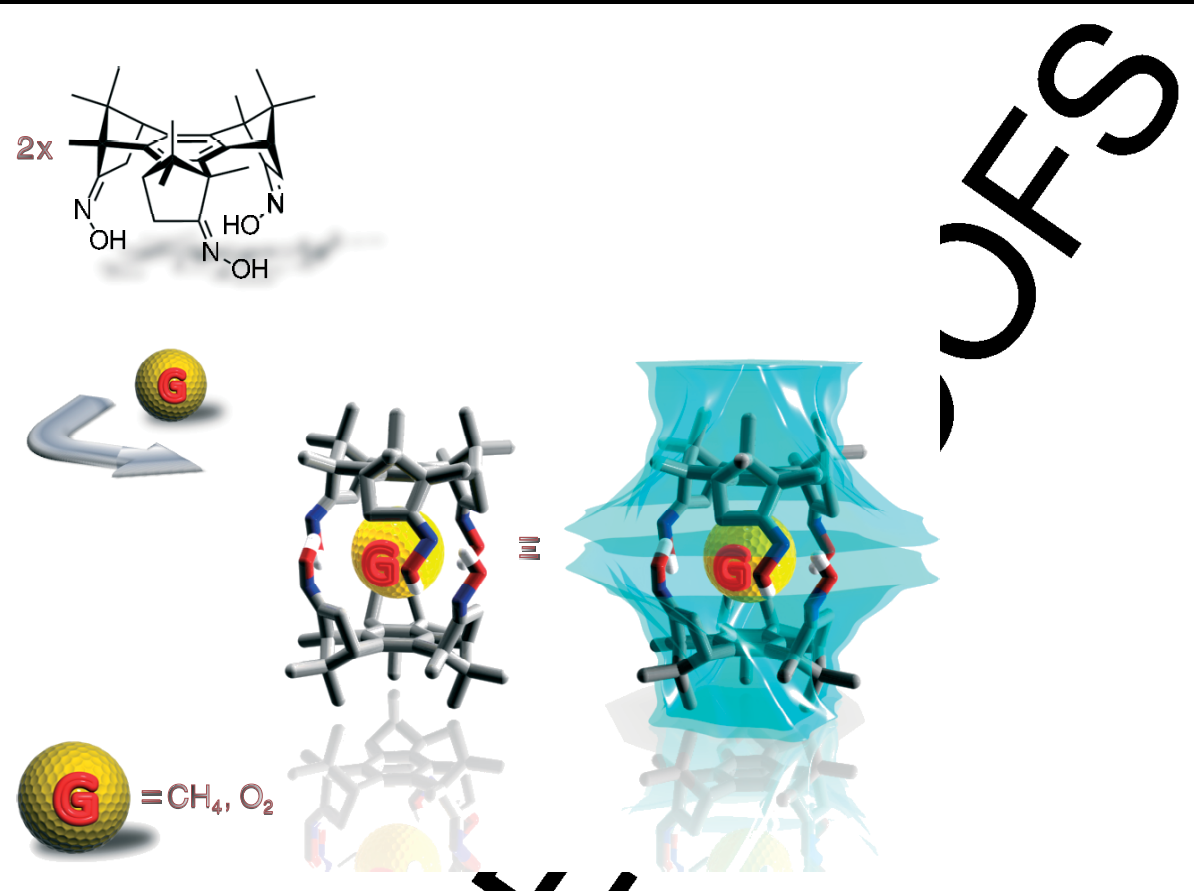

Figure 2 Self-assembling dimerization of the benzocyclotrim tris-o leading to molecular capsule for methane and oxygen hosting.

connected to the aromatic spacer, ${ }^{16}$ or the syste $n$ was endowed with amino groups on the side chains of the glycoluril giving rise to enhanced solubility lation of argon and xenon, but also the reve sible control over the association-dissociation process by in of $s$ dition of $p$-toluensulfonic acid or sodium en nato 7 Even heterodimeric capsules were prepared oy s and mixing monomers characterized by different spa like ethylene, benzene, and naphthalene etheno attr ace confirming the high dimerization ability of te gl con residue and the good complementarity provido by the curvature of the monomers forming hybr cap les with disproportion equilibria driven by the ef ploymer of sized solvents as guests. $^{18}$

\subsubsection{Tris-oxime d ner}

While there are seve sysums based on glycoluril or urea hydrogen-bon for the preparation of dimeric self-assembled o ing to their intrinsic attitude to the formation of actme interactions, much less has been investig in the development of new hydrogen-bonding functional g wans hat are able to provide strong and directional interactions. Recently, Scarso and Fabris reported the preparation of a chiral benzocyclotrimeric system adorned with three oxime residues that self-assembled in chloroform- $d$, forming a capsule characterized by a cavity of about $46 \AA{ }^{3}$ which accommodates molecular oxygen under fast exchange on the NMR timescale as confirmed by the broadening of the resonances of the capsule (Figure 2). ${ }^{19}$ This phenomenon is ascribable to the paramagnetic properties of molecular oxygen that cause the relaxation of nuclei positioned in proximity, thus acting as a probe molecule for determining its position in the equatorial portion of the cavity. Bubbling of inert gases like $\mathrm{N}_{2}$ or $\mathrm{CH}_{4}$ leads to sharp signals and encapsulation of the incoming gaseous guests, as confirmed at low temperature where exchange processes slowed down showing clear upfield signals for the encapsulated methane.

\subsubsection{Tris(ureidobenzyl)amine}

Self-assembling capsules are usually based on conformationally restricted systems in order to minimize entropy losses; therefore, aromatic surfaces connected by not more than one atom are preferred over flexible linear segments. Rigidity and preorganization favor self-recognition, but a certain degree of flexibility would allow the preparation of systems that more closely resemble biological receptors where flexibility and adaptation of the host to complement the guest are commonly observed. One of the dimeric self-assembling capsules characterized by flexible hydrogen bonding units is based on tris(ureidobenzyl)amines ${ }^{20}$ with substitution in position 2 or 3 of the aromatic residue leading to monomeric species that show $C_{3 \mathrm{v}}$ symmetry in polar solvents such as (dimethyl sulfoxide) DMSO- $d_{6}$ but 

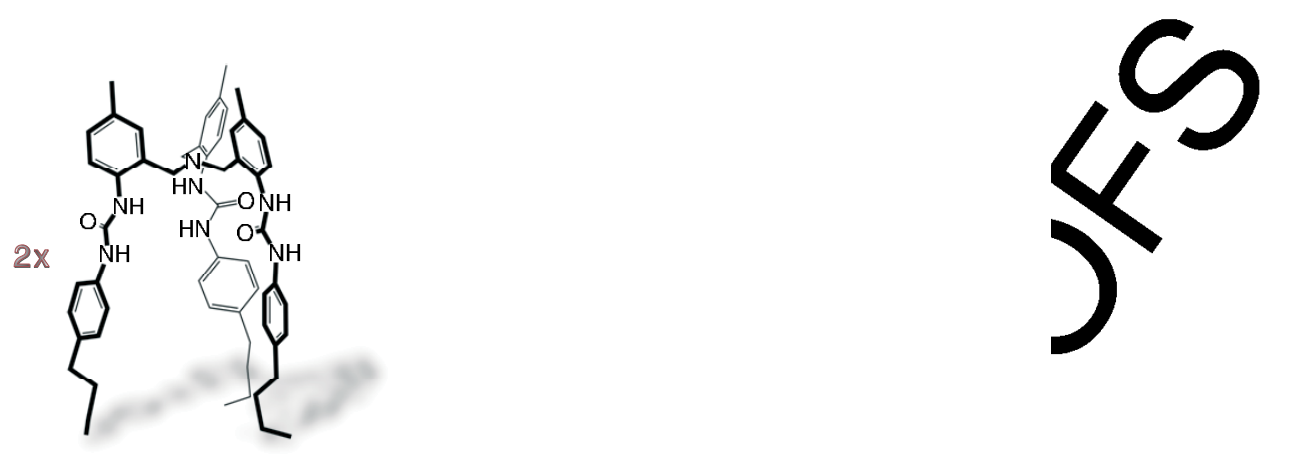

\section{5}
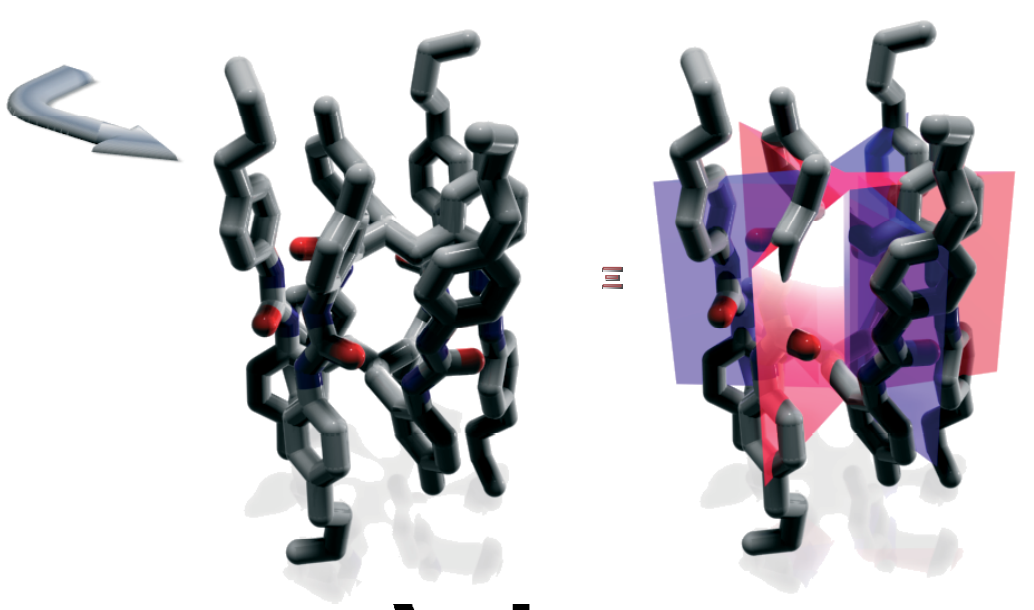

Figure 3 Homodimerization of urea-based tris(2The larger tris(3-ureidobenzyl)amine showed the

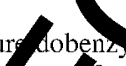

ne, forming a capsule that is very small to accommodate guests. ence of one encapsulated solvent molecule.

form dimeric capsular assemblies in chlo and soments in slow exchange between monomer d d ner on the NMR timescale. In particular, tris(2-ureto senzyl)amine shows formation of enthalpically dr en imerio capsule ${ }^{21}$ via interplay of six hydrogen-bont $y$ eas with no guest included because of the small anace ailable and with blocked conformation of the romatio residues in a chiralpropeller-like structure with a overall symmetry derived by one clockwise and the herclockwise arrangement of the tris(benzyl (mino r idues (Figure 3).

The larger tris(3-ure tobenzy) amine forms analogous dimeric capsules the shace to accommodate one molecule of mes. which could be $\mathrm{CH}_{3} \mathrm{NO}_{2}$, dichloromethane, $\mathrm{CH}_{3} \mathrm{I}$ in increasing order of affinity, using toluene- $\lambda_{8}$ a a competitive solvent observing fast in-out of the gues at room temperature and requiring a temperature low as $213 \mathrm{~K}$ to move to slow exchange observing separate resonances for free and bound guest. ${ }^{22,23}$ Using chiral racemic tris(3-ureidobenzyl)amine ${ }^{24}$ characterized by the presence of a stereocenter in one arm of the tris(benzyl)amine led to the preferential formation in solution of only two isomeric capsules out of the seven possible if considering the reciprocal position of the stereocenters of the two subunits; all driven by a point-to-helix chirality transfer, which means that the asymmetry present in the stereocenter steers the asymmetry of the helical assembly.

\subsubsection{Calix/47urea}

The urea moiety provides a good combination of hydrogen bond donor and acceptor character in the same functional group, which can be implemented on different molecular scaffolds leading to self-assembling systems. Calix[4]arene scaffold is characterized by a semirigid concave surface and, if decorated with urea moiety on every aromatic residue, forms dimeric capsules with the shape of two facing square pyramids reciprocally rotated by about $45^{\circ}$ toward each other. Tight binding between two units is ensured by a seam of 16 hydrogen bonds derived by interdigitating alternating urea moieties that, behaving like a zip, seal the equatorial region of the supramolecular structure forming a cavity of about $200 \AA^{3}$ in a quantitative way for concentrations above micromoles. ${ }^{25,26}$ Such a cavity can host neutral solvent molecules with affinity in the order $p$-xylene $<$ toluene $<$ benzene $\approx$ chloroform $^{27}$ as well as 
fluorobenzene, all in slow exchange on the NMR timescale. Alternative guests are cationic species like tetralkylammonium species of congruent sizes and shapes that can experience attractive cation $-\pi$ contacts with the inner aromatic surfaces of the capsule. Hydrogen-bonded dimeric capsules based on the same calix[4]arene scaffold but endowed with different hydrogen bonding tags like tetraureidopeptide $^{28}$ moieties provided slightly larger capsules where extra hydrogen-bonding contacts were present.

Combinations of equimolar amounts of tetraarylurea and tetratosylurea calix[4]arenes curiously led to the exclusive formation of heterodimeric capsules (Figure 4). The presence of different halves in combination with clockwise and counterclockwise head-to-tail arrangement of the hydrogen bond seam makes the assembly chiral ${ }^{29}$ showing diastereoselective binding of chiral enantiopure guests like $(+)$-nopinone or $(-)$-myrtenal. Reasons for such specific heterodimerization processes are still un er oa plausible explanations are the good match between $t$ te more acidic hydrogen-bond donor tosylur combinea with the more basic hydrogen bond accepto ea and the fact that tetratosylurea dimers are chnorerny by conformations that are sensibly higher (energy rompared to those assumed in the heterodimers. ${ }^{3}$ This cla , of heterodimeric capsules is the smallest ondonolecular aggregate that has displayed enan oselect binding. Interaction of a racemic guest charact ized by rigid bicyclic structure like norcamphor with te fin capsule resulted in the formation of two ets diastereoisomeric assemblies in a $1.3: 1$ ratio ((dro coisomeric effect) d.e. $13 \%){ }^{31}$ The same level of reos rectivity was observed using $(R)$ (+)-3-methy cyclo dentanone as an enantiopure guest that is selectively and only on the basis of steric interactions, indicating the in order to achieve higher selectivities,

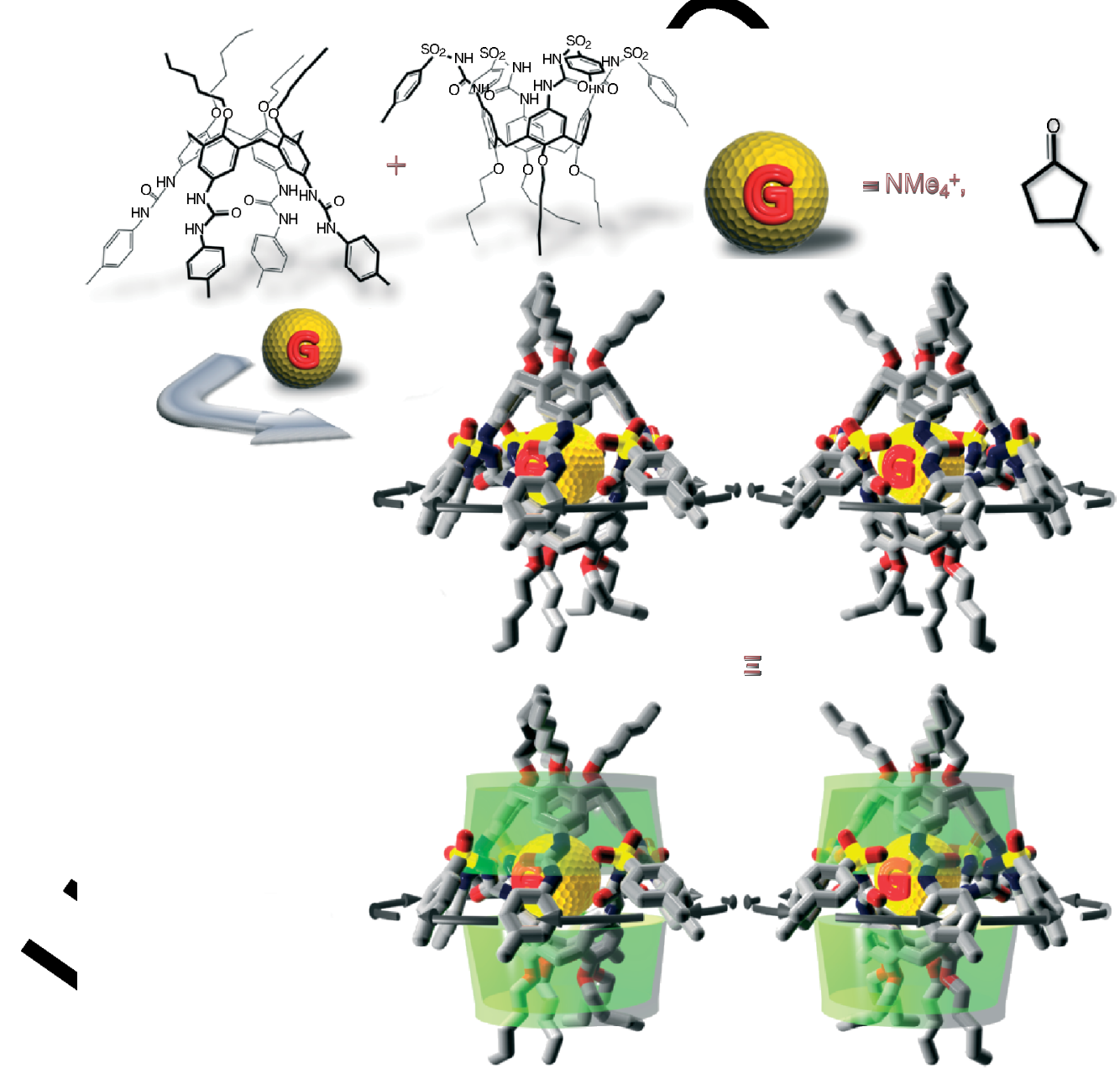

Figure 4 Preferential heterodimerization between tetraarylurea and tetratosylurea calix[4]arenes leading to supramolecularly chiral capsules that can host cationic or neutral guests. 

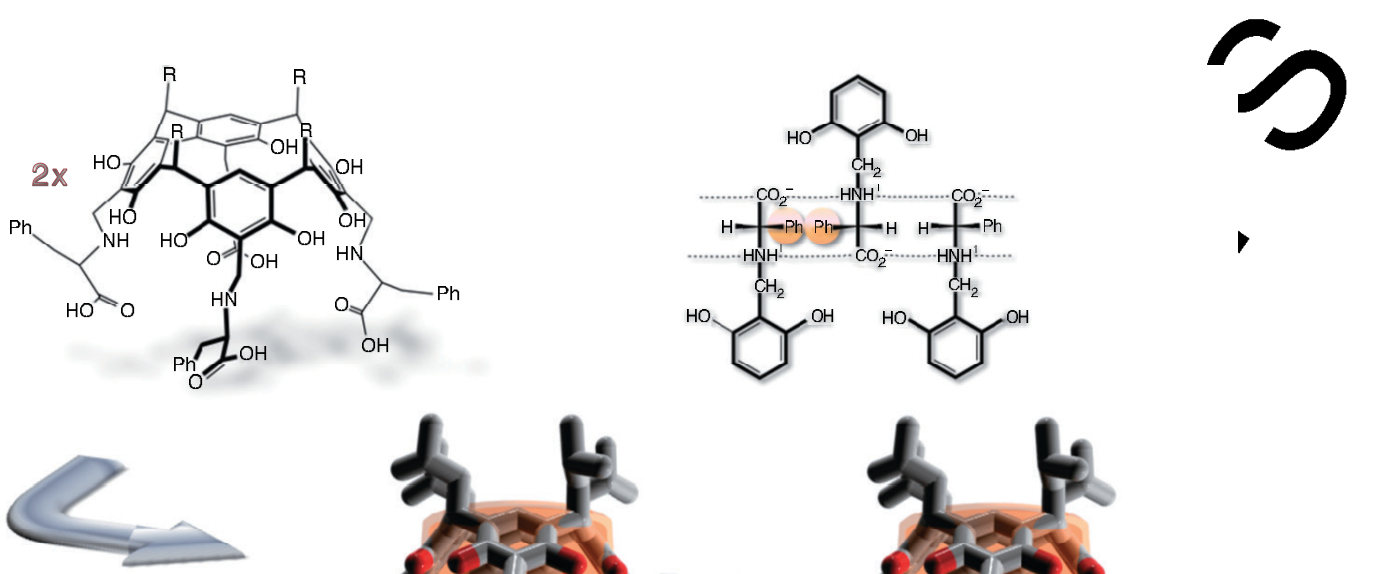

$=\mathrm{H}_{2} \mathrm{O}, \mathrm{CH}_{3} \mathrm{NO}_{2}$
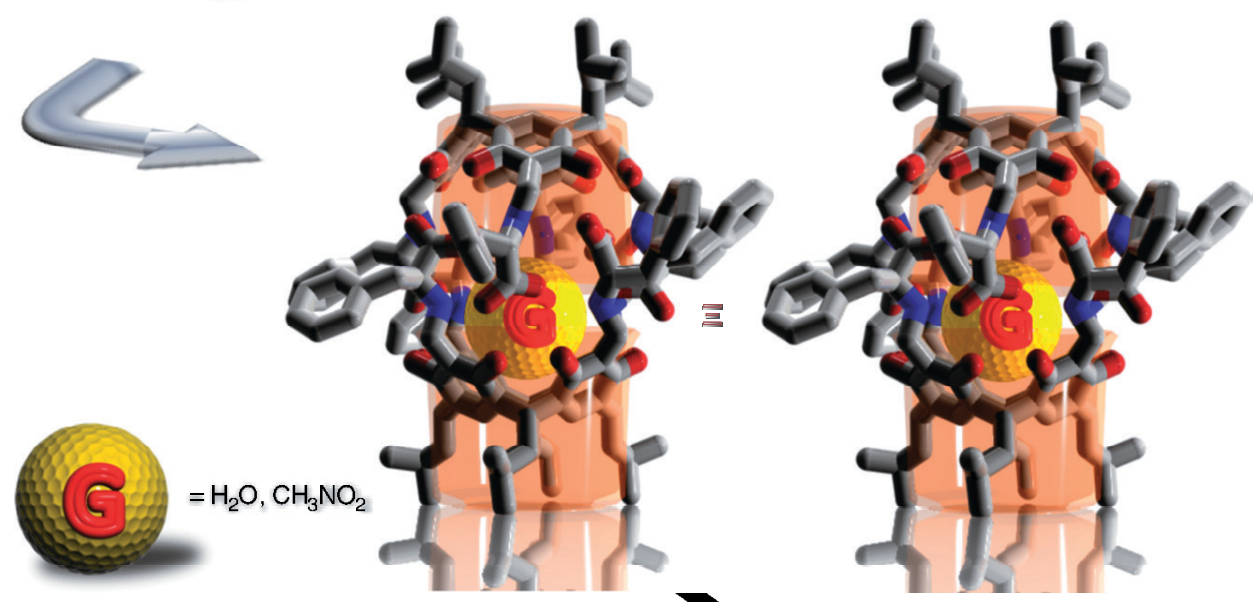

Figure 5 Homodimeric capsule assembled via a seam of narged lydrogen bonds between phenylalanine residues in apolar solvents.

closer contacts between the host and guest a via attractive weak interactions. The exclusive ization process has been exploited for the of a large variability of functional molec capsular polymers, ${ }^{25}$ dendrimers, ${ }^{26}$ and han are beyond the scope of the present conth fion. Several permutations on the kind of substi an present on the urea moieties for homodimeric cap 1 les $10 \mathrm{mel}$ to observe supramolecular chirality only due to te mutual arrangement of the two achiral calix atrent in se dimer. ${ }^{32}$

\subsubsection{N-linked phenylonth roin[4]arene}

Tighter association onstants were observed using resorcin[4]arene-based ructurs endowed with four $N$-linked phenylalan residues that enable the self-dimerization via formation of two seams of salt hrids formed between the amine and the carboxylic group suo a capsule is highly kinetically stable, wi a cavit of about $310 \AA^{3}$ large enough for coencapsulation offferent guests that, contrary to what is commonly observed, need to be polar like nitromethane, alcohols, and, quite surprisingly, also water owing to the strong interactions via hydrogen bonding with the polar equatorial belt of the capsule under slow exchange on the NMR timescale (Figure 5). ${ }^{33,34}$ In particular, because of the intrinsic chirality of the capsule, diastereoselective binding of chiral hydroxy acids was possible with d.e. up to $59 \%$ for coencapsulation of such guests together with one molecule of acetone- $d_{6}$ or water. ${ }^{35}$

Similar strong hydrogen bonding between polar charged groups allowed dimerization in competitive solvents as observed for amino-calyx[4]arene systems connected through the $\mathrm{C}$-terminus to four alanine residues. Such polar cavitand self-assemble in methanol- $d_{4}$ containing $4 \%$ of $\mathrm{D}_{2} \mathrm{O}$ as a polar solvent by means of a seam of hydrogen bonds between protonated terminal amino groups and carbonyl residues with an association constant of $29000 \mathrm{M}^{-1} .36$ This system represents a connection between uncharged capsules in apolar solvents and purely ionic capsules in polar solvents.

\subsubsection{Tetraurea-calix[4]pyrrole}

Orientation of guests within self-assembled capsules is usually driven by the size and shape selectivity as most of the subunits employed lack the presence of inwardly oriented functional groups that can bind the guest weekly and reversibly. Exceptions to this were discovered recently by the group of Ballester that reported the preparation of dimeric capsules based on the installation of urea functions in the para position of the $\alpha, \alpha, \alpha, \alpha$-stereoisomer 

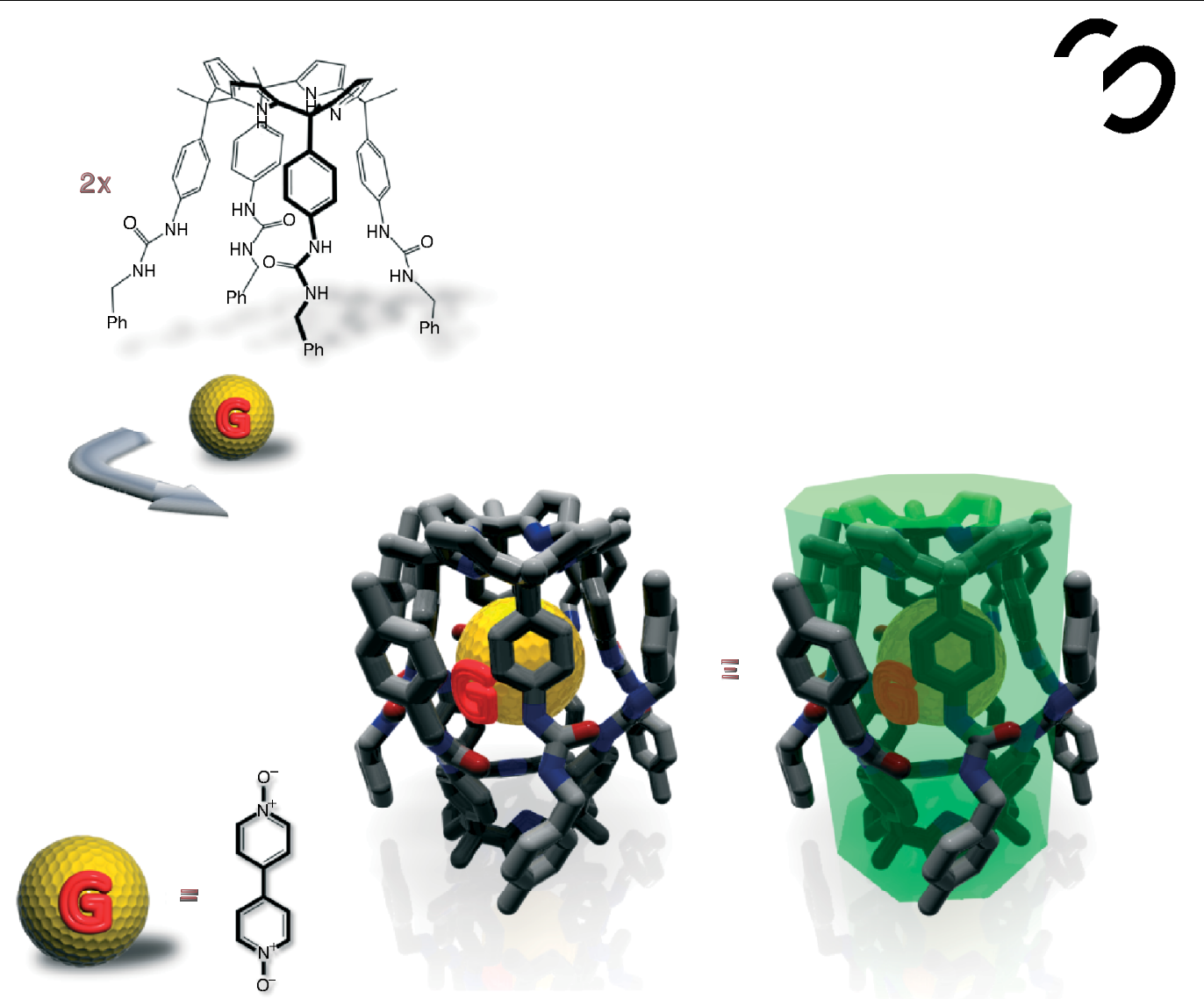

Figure 6 Homodimeric capsule formed by hydros bond $g$ between urea moieties and guest encapsulation via attractive hydrogen bonding between the guest and the calix[ 4

of meso-tetraphenyl-tetramethyl-substitun calix[4]pyrrole (Figure 6)..$^{37}$

The monomer presents urea bieti othe dimerization process and pyrrole NH resid. that converge in the calix[4]pyrrole structure pro curme unta hydrogen bond donors for suitable guests endowed with hydrogen bond acceptors at both extremitio like 4 4 -pyridine bis- $N, N^{\prime}$ oxide ${ }^{38}$ In fact, the ch ax 4$]$ pyrure does not provide clear solutions in $\mathrm{CD}_{2} \mathrm{Cl}_{2}$ lt clear gnals appear upon addition of half equivalent of pyri ne bis- $N, N^{\prime}$-oxide that has the correct size and ape to fill about half of the volume of the cavity th a PC close to ideality and that makes stron hy agen-bond contacts with the electronrich oxygen at os the calix[4]pyrrole top and bottom of the vsule. ${ }^{6}$ haller guests can be used but necessitate the pres of good hydrogen bond acceptor moieties like for trimethyl phosphine oxide and trimethyl ammine oxide that can be coencapsulated in the dimeric capsule, both oriented with the oxygen atoms toward the pyrrole residues. The head-to-tail directionality of the urea groups can be clockwise or counterclockwise and interconversion is slow enough with an activation barrier of $16.6 \mathrm{kcal} \mathrm{mol}^{-1}$ for the change of direction of the urea belt that makes chiral the dimer. When using chiral tetraurea calix[4]pyrrole scaffolds bearing stereogenic centers close to the urea residues, the dimeric capsules obtained with suitable guests acquire another element of asymmetry due to the $M$ or $P$ chirality induced by the urea belt. Overall, it is worth noting that, analogous to that observed with tetraureidocalix[4]arenes and tetraureido-calix[4]pyrroles, while enantiopure $(S)$ monomer self-assemble into $(S, P \cdot S, M)$ dimer, when racemic tetraurea is used, there is preferential formation of achiral meso- $(S, P \cdot R, M)$ hetero dimer. ${ }^{39}$

\subsubsection{Softball}

Glycoluril subunits, as versatile hydrogen bonding units, have been implemented into several polycyclic rigid structures bearing bridged bicyclic centerpiece giving rise to many dimeric pseudospherical aggregates generally known as "softballs." Here the cavity is rather large (from 200 to $400 \AA^{3}$ ) and up to two guests can be accommodated 

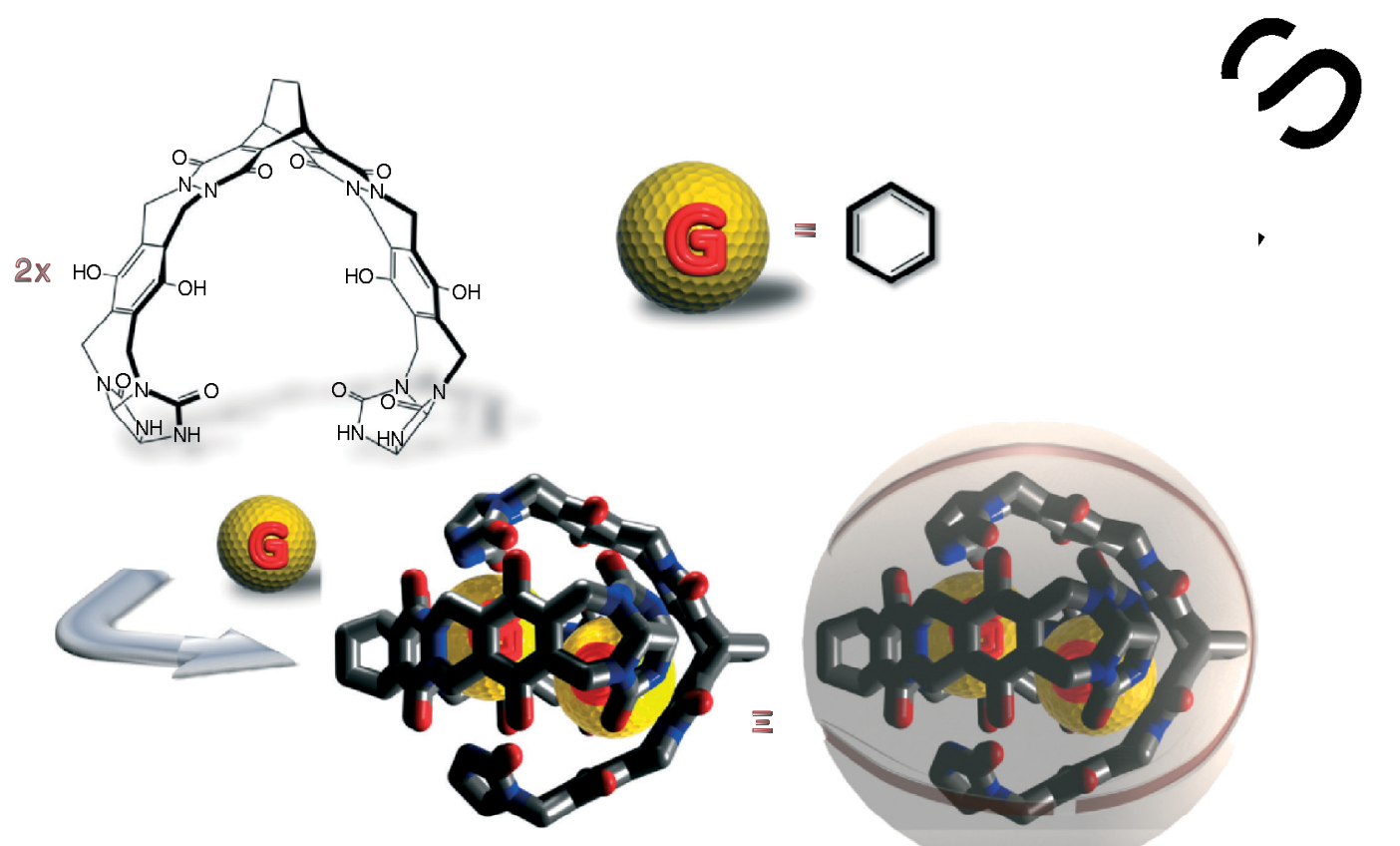

目<smiles>c1ccccc1</smiles>

,

Figure 7 Homodimeric softball capsule hosting two molecule of bo zene as guests.

(Figure 7). ${ }^{40}$ These kind of capsules have found inter sting applications in several fields; two in particular, mentioning. The first is the synthesis of chiral possible, one based on the employment of liffen 1 - 1 coluril residues at the extremities of a sym netri -pacer ${ }^{41}$ and the other based on the same glycolm acture connected to spacers bearing different bicy un un both the cases, the monomers are achiral but e di escapsules are chiral as a typical example of suprar cular chirality.,42

Addition of chiral enantiop class of chiral softballs led to the frmation of two diastereoisomeric host-guest systems, un grtunate $y$ in equal amounts because the cavity was ity of the guest. To pro fide ser tivity in the binding due to stereorecognition cl er inte actions and tighter binding are pivotal requis s, and the second kind of chiral softballs achieve In this case, the dissymmetric inner surface on the cavity easily sensed chiral guests, providing differo dianereoisomeric complexes in different amoun Each a stereoisomer is characterized by four different NH son hes because of the complete loss of symmetry of the complex. A total of six different glycoluril monomers with overall twenty 20 chiral enantiopure guests were investigated ${ }^{44}$ observing that the presence of functional groups capable of hydrogen bonding to the host was crucial for affinity as well as selectivity. In a few cases, the selectivity allowed d.e. above $50 \%$ and never more than $60 \%$ probably because the average distance between the capsule and the chiral content is not optimized and, even in the presence of a good fit and high PC; about half of the cavity's volume is still not filled and this space surrounds the guests. 6

The high kinetic stability of softballs, which dissociates and therefore racemizes with rates much smaller compared to the in-out exchange of the guest, allowed the demonstration of the chiral memory effect concept (Figure 8). It was possible to template the formation of a chiral enantiopure capsule with a chiral enantiopure guest like $(+)$-pinanediol favoring one of the two enantiomeric host structures, then the guest was replaced by an achiral one like leaving a chiral nonracemic host capsule that slowly reequilibrates to the thermodynamically racemic mixture. ${ }^{45}$

The second important application of softballs is their use as supramolecular catalysts in the Diels-Alder reactions. ${ }^{46}$ In particular, after careful substrate selection, the system is optimized and true turnover ability is possible for the reaction of $p$-quinone and thiophene dioxide (Figure 9). The reaction is spontaneous in solution and provides 10 and $17 \%$ of cycloaddition products after two and four days respectively. With softball as catalyst, 55 and $75 \%$ conversion was achieved after the same time periods. ${ }^{47}$ More importantly, the capsule provided true turnover because of the higher steric clashes of the product compared to the 
10 Self-processes
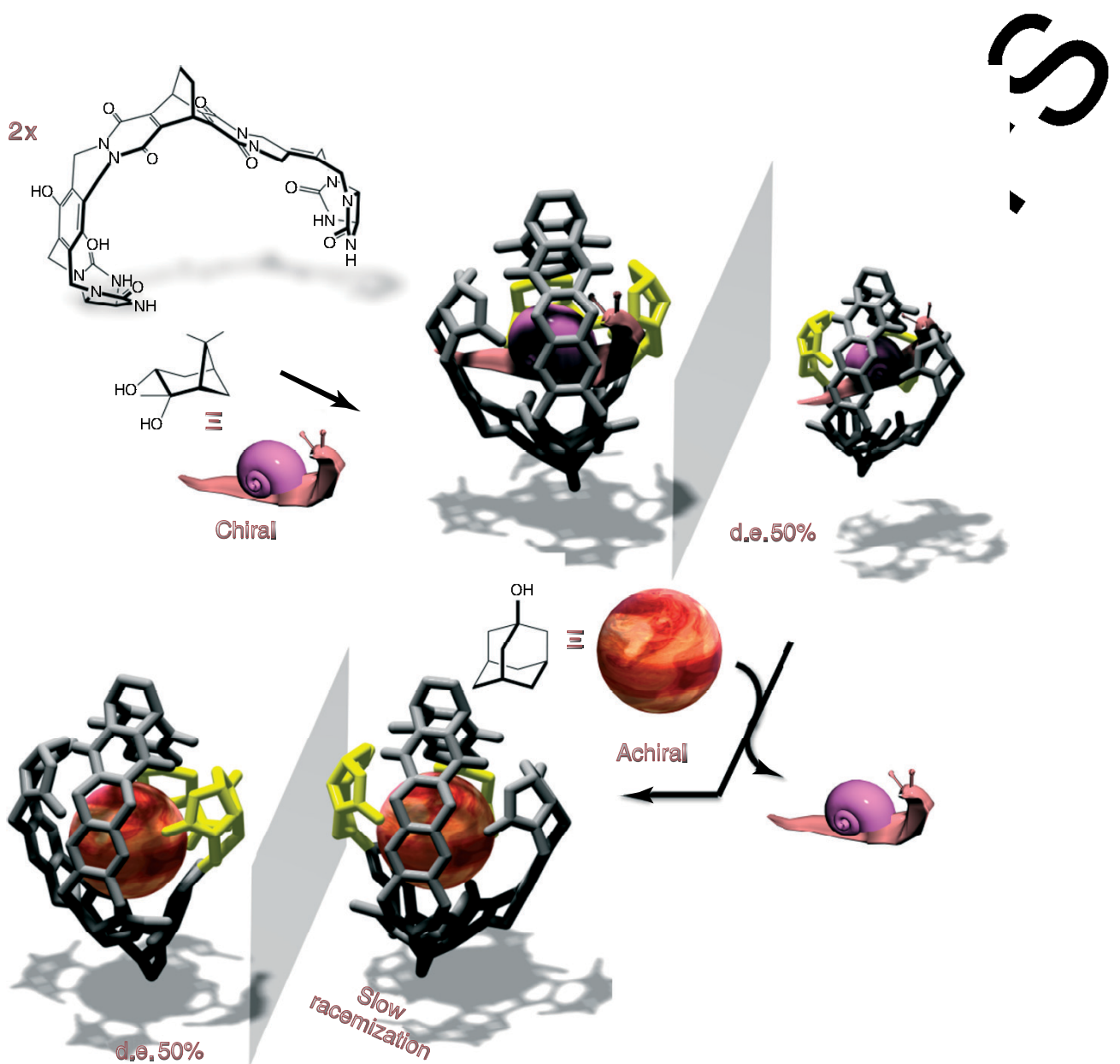

Figure 8 Chiral memory effect displayed fball capsule templated with a chiral guest that is subsequently replaced by an achiral one, leading to an enantiomerically enring soll that slowizes.

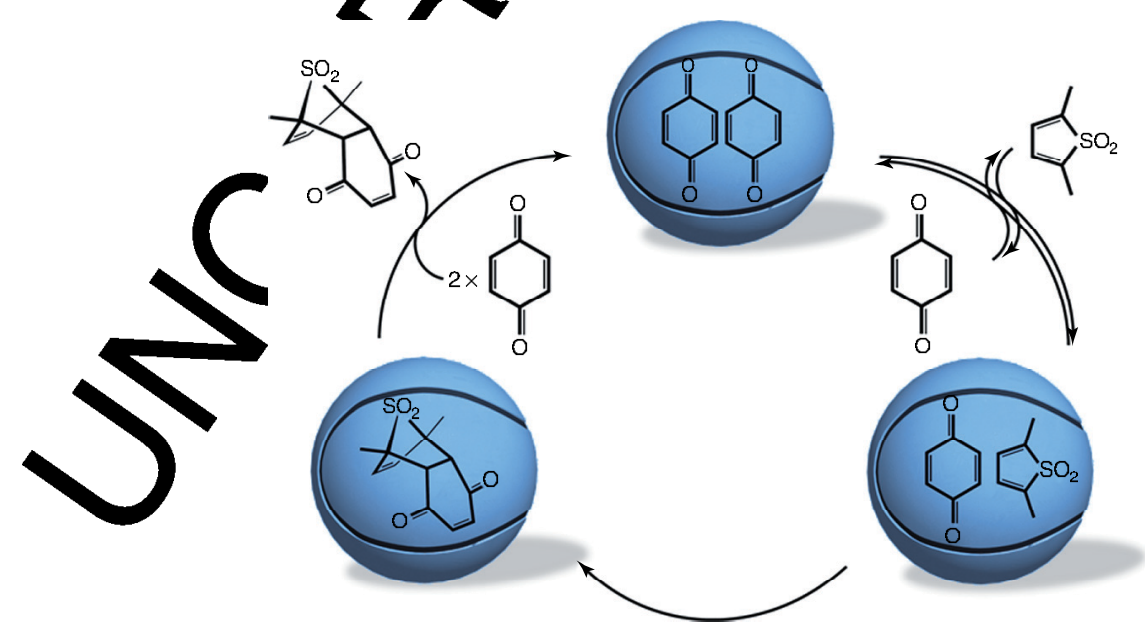

Figure 9 Catalytic Diels-Alder reaction mediated by the hydrogen-bonded softball capsule. 
coencapsulation of two molecules of $p$-quinone, which was the resting state of the catalyst in the cycle.

\subsubsection{Cylindrical capsule}

Most of the hydrogen-bonded capsules are based in rather shallow cavitands adorned with hydrogen-bonding units on the rim. This leads to the formation of aggregates with a shape that can span from pseudospherical to egg shaped as a function of the depth of the cavitand employed and on the difference between the diameter of the aperture and the subunits employed. Only one example is known of a cylindrical capsule formed by dimerization of a deep cavitand. The latter features a resorcin[4]arene base to which four aromatic walls with imide functionalities on the top are attached. Two modules self-assemble through a network of eight bifurcated hydrogen bonds and result in a cavity of about $425 \AA^{3}$ that can be filled with a very large combination of up to three guest molecules, ${ }^{48}$ with preference for neutral or anionic species (Figure 10). Usually the solvent of choice for this kind of studies is mesitylene- $d_{12}$ because its size and shape hamper the encapsulation, leaving access to the cavity for other guest present in the solution even at a rather low concentration

\section{mole}

\section{(1)}
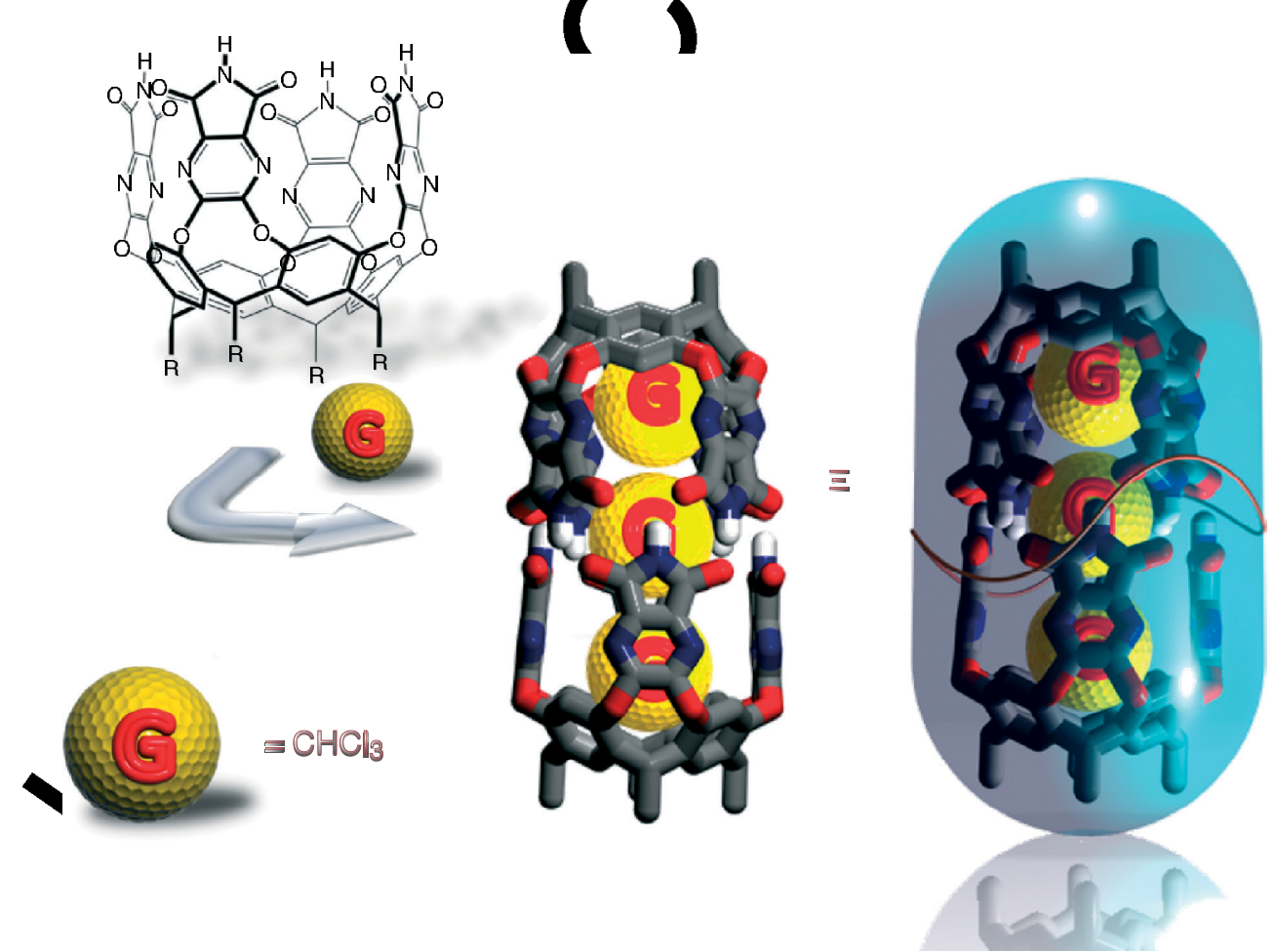

Figure 10 Cylindrical homodimeric capsule featuring an elongated cavity that can accommodate up to three guest molecules. 
the keto and enol form of the latter species within the capsule depends on the kind of second guest, while in the bulk solvent the two do not interact with each other and the keto-enol ratio is insensitive to the nature of the cosolvent. ${ }^{52}$ Using $p$-cyclophane as the primary guest that perfectly fits the square section of the cavity, it was observed that the size of a second coencapsulated guest can finely regulate the spinning rate of the first, also on the basis of steric interactions. ${ }^{53}$ In the presence of rather large guests, it was possible to coencapsulate different gaseous guests like $p$-xylene- $d_{10}$ with ethane or anthracene with methane in the residual cavity. ${ }^{54}$

In unimolecular guests, the kinetic stability of the ternary aggregate increases and the system withstands the presence of small amounts of protic solvents.$^{55}$ The cavity can accommodate long alkanes from decane through tetradecane, (Figure 11) but, while the former is encapsulated in its extended conformation, the latter is too long and enters the cavity adopting several gauche conformations assuming a helical shape that permits better $\mathrm{CH}-\pi$ contacts between the guest and the aromatic surfaces of the capsule, thus compensating the extra energy required for folding. ${ }^{56,57}$

This kind of capsule was used to prove concepts related to chiral sensing and chiral induction across the space, b between species coencapsulated within the cavity and also between guests and chiral residues outside the cap ale. In the first case, combinations of two molecules of chiral racemic species led to diastereoisomeric host-g est semblies like in the cases of $( \pm$-trans-1,2-cyclol exan diol; $( \pm)$ - $\alpha$-halo, or $( \pm)$ - $\alpha$-hydroxy acids ${ }^{59}$ with set eose $c$ tive ratios up to $1.6: 1$. Concomitant ensulo also allows the reciprocal interaction betwee two morent chiral guests, for example, the stronger hy an bond donor

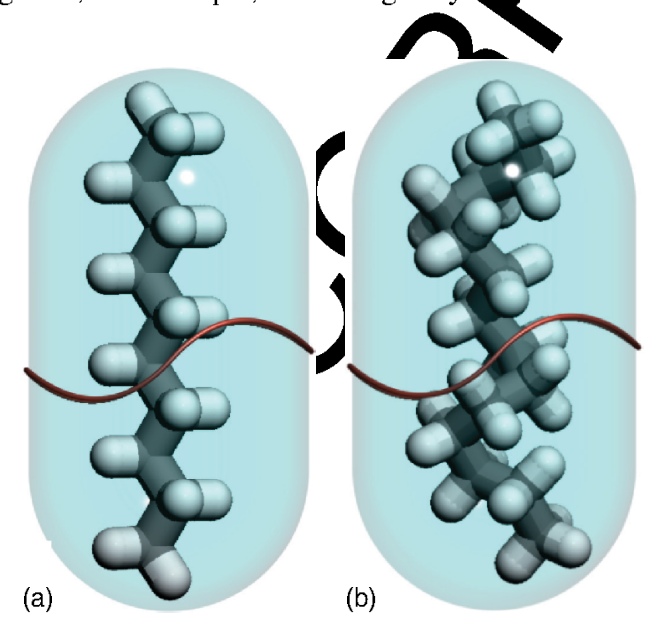

Figure 11 (a) $n$-decane encapsulated in the cylindrical capsule in an extended conformation; (b) $n$-tetradecane is too long to fit the cavity and it is encapsulated only assuming a shorter helical confirmation. like $(S)$-mandelic acid with ( \pm )-2-butanol (ivin to to two diasteroisomeric complexes, this time the ratio $3: 1$ at $283 \mathrm{~K}$, or between $(S)$-1-phenylethar 1 ar (19-3-methyl2-bromo-butyric acid with a $1.5: 1$ reoselective ratio because of the presence of attro in guest hydrogen bonds that are instructed and fored $b$, the shape and size of the cavity of the molecular apsule.

In the second case, a isopropanol was coencapsulated with $m s o-1,1$ enzene-1,4-diyldiethanol containing one $R$ and de $S$ ste cocenter with $\left(1 R, 1^{\prime} R\right)$ 1,1'-benzene-1,4-diy than th both stereocenters with the same configur con. signals of the methyl groups of isopropanol were creotopic because of the chiral environment creat an tho yrger guest, but more importantly the differen in hemical shift between each couple of doublets was $1 \mathrm{ppm}$ with the meso guest and 0.27 with the enantiopure gust. This low but significant difference impli uto the isopropanol senses not only the asymmetry imp rted by a stereocenter close to it but also the other streonter fore than $10 \AA$ away facing the bottom of the capsyle. T.us phenomenon is not related to distortions or ve c araments of the hosting structure and is ascribable to atic asymmetry. ${ }^{61} \mathrm{~A}$ further step was disclosed on long-range chiral effects using cylindrical capsules bearing rreocenters on the alkyl chains of the resorcin[4]arene scaffold observing their effect from the outside of the capsule directly on the guest trapped in the inside. Using a biphenyl guest bearing an isopropyl ester moiety encapsulated in chiral footed capsules, it was clearly observed that the resonances of the isopropyl residue were diastereotopic as a consequence of the chirality of the host that induces its magnetic effects through the capsule to the bound guest. ${ }^{62}$

Last but not the least, this kind of capsule has been largely employment as a nanometric reaction chamber for a vast range of reversible and irreversible chemical reactions with the aim of underlining the effect of encapsulation phenomena on product selectivity. Correct size and shape complementarity is crucial for the catalysis of bimolecular reactions with capsules. The cylindrical capsule preferred the coencapsulation of phenyl azide with phenylacetylene with both the aromatic residues near the tapered ends of the capsule leading to 1,3-dipolar cycloaddition reaction with specific formation of the 1,4-triazole isomeric product, while in solution both isomeric 1,4 and 1,5 products were observed in comparable amounts (Figure 12a). ${ }^{63}$ Another example of product selectivity comes from the reaction between carboxylic acids and isonitriles, ${ }^{64}$ which, when performed in the bulk solvent leads to the formamide derivative together with anhydride, while, on the contrary, when run within the cylindrical capsule the main species detected in the cavity is a rearrangement product $N$-butyl$\mathrm{N}$-formyl-2-(4-methylphenyl)acetamide (Figure 12b) as a consequence of the reciprocal orientation of the substrates 

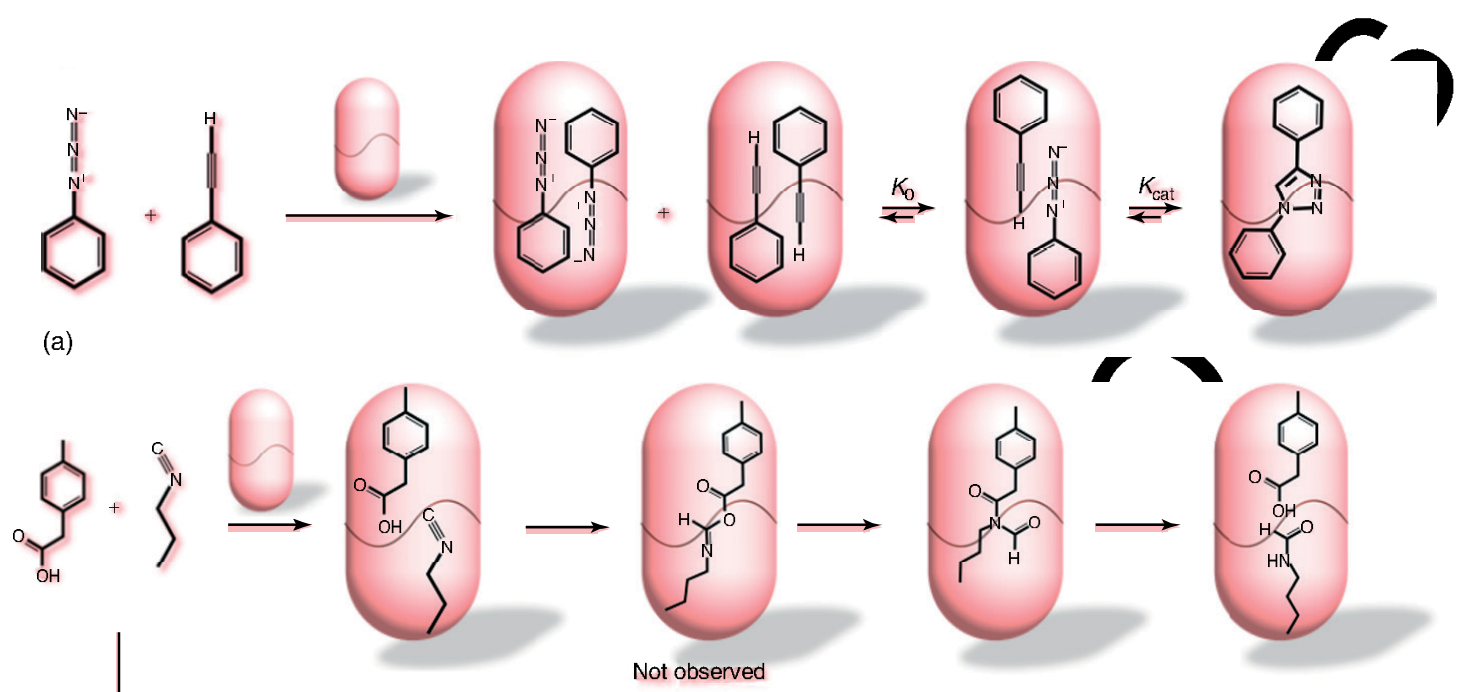

(b)

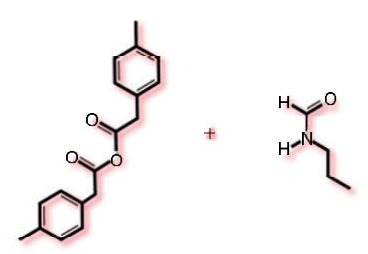

Figure 12 Examples of product selectivity driven by the dimerh cylindrical capsule: (a) reaction between phenylazide and phenylacetylene; (b) reaction between carboxylic acid and

in the cavity with polar residues allocated toward tbo mile of the capsule where the seam of the hydrogen and acceptor can stabilize polar transition stat

\subsection{Multimeric capsules}

\subsubsection{Tetrameric capsule}

Tetrameric capsules are much less amon; one known example is characterized by an we head-to-tail orientation of the hydrogen-bo ding m nomers forming a barrel-like capsule with a pse to-sphe cal cavity of about $160-184 \AA .^{3}$ The mono ers are vased on a rigid aromatic spacer with a sulfamid moiety a typical good hydrogen bond donor) on one idd and a ycoluril residue (a typical good hydrogen bond ao story on the other, while positions 1 and 4 presen atom, ${ }^{65} \mathrm{OH}^{66}$ (Figure 13) or $\mathrm{H}, \mathrm{OCH}_{3}{ }^{62}$ res yes when the spacer is a naphthyl residue. The mon mes are scarcely soluble in noncompetitive suents an, only upon addition of a suitable guest, they an a $/ 1$ a tetrameric capsular form surrounding the guest that can make good $\mathrm{CH}-\pi$ and van deer Waals contacts with the aromatic surfaces of the aggregate. In particular, the smaller subunit binds pseudospherical guests like adamantane in dichloromethane- $d_{2}$ with a remarkable entropic loss due to the multimeric nature of the capsule that is overcome by good fitting of the cavity and positive attractive contacts between the surfaces of the host and the guest. Even better binding was possible with 2-adamantanone and 2,6-adamantanedione where the carbonyl residue offers extra attractive interactions via hydrogen bonding with polar ends of the capsule, giving rise to extremely good fitting of the cavity and high PC up to $72 \%$. The longer representative of this family of capsules showed impressive differences in binding affinity as a function of the functional groups present on the central aromatic core. The methoxy-substituted monomer self-assembles in the presence of suitable guests like polycyclic molecules such as 1,3,5,7-tetramethyladamantane, camphor, or congressane, while the H-substituted analog does not, even if it seems to be less sterically hindered. Explanation for such behavior arises from more intense hydrogen bonding in the former system caused by the electronic effect of the methoxy group that can also make favorable van deer Waals contacts with the neighboring aromatic rings. If the monomer is substituted with only one $\mathrm{OH}$ group in the aromatic central core, the molecule is chiral, and if used in the enantiopure form it provides an enantiopure tetrameric capsule displaying diastereoselection in the encapsulation of racemic guests. ${ }^{68}$ In particular, ketone molecules showed the highest affinity and chiral recognition depending more on the positioning of functional groups 


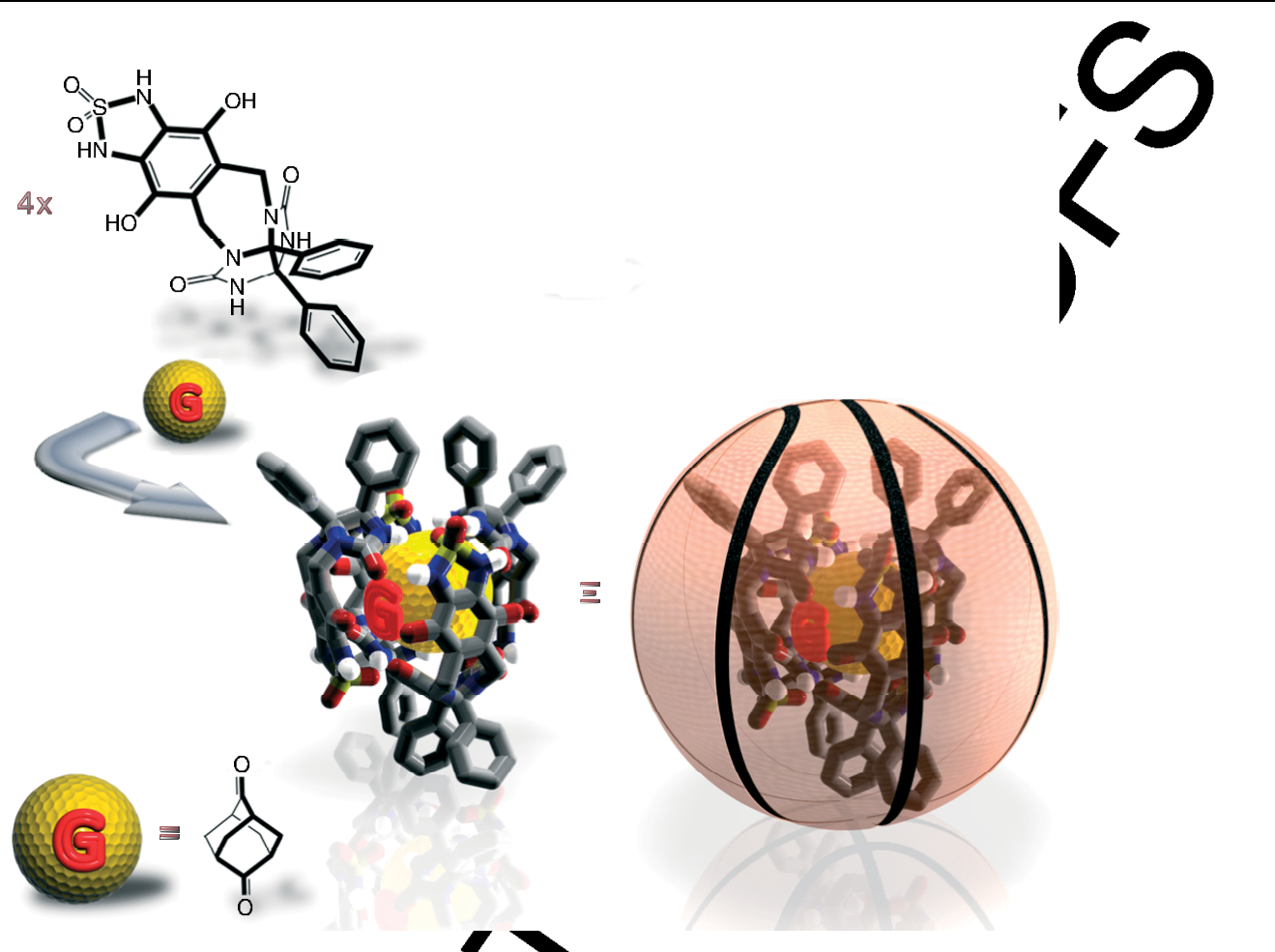

Figure 13 Tetrameric capsule showing binding of suitablests thathake multipoint hydrogen bonding with the top and bottom of the capsule. in the capsule rather than the size of the gues as grerved
for $( \pm)$-norbornadione and $( \pm)$-3-methylcyclo has almost the same molecular volume boshow of 23 and $60 \%$ respectively.

\subsubsection{Hexameric resorcin $/ 4$ pyrogallo[4]arene}

The largest known hydroge oor ed pramolecular capsule is based on self-assem ly of on of the most used and easy to prepare scaffold: re $\operatorname{rcin}[4]$ ene. This molecule is characterized by a cur eurshaponparted by the four aromatic residues and it fresents ight hydroxyl residues that adorn the rim of the sh low co itand. In 1997, Atwood first reported the resorcin arenc self-assembly behavior both in the solid state rolution in benzene- $d_{6}$, evidencing the forpation of pseudospherical capsule comprising six units held netho through a seam of about 60 hydrogen bor betwe phenol groups and water molecules (Figure 14). In restingly, the hexameric capsule is chiral because of the twisted positioning of the calix[4]arenes with respect to the eight water molecules arranged in a cubic fashion. The assembly exists in organic solvents like chloroform- $d$ or benzene- $d_{6}$ even at concentrations as low as nanomolar, ${ }^{70,71}$ but only if the solvent is saturated with water. The cavity is about $1375 \AA^{3}$ and such a space is usually filled by six to eight solvent molecules. Suitable guests are usually cationic species ${ }^{72}$ like tetraalkylammonium and phosphonium cations that, depending on their size, are coencapsulated with residual solvent molecules. Trapped guests show upfield NMR signals separated from those of the free guests in the bulk because in-out exchange is a slow process on the NMR as ingress and egress of guests occurs likely via temporary dissociation of one subunit from the hexameric structure. ${ }^{73}$ Other common guests are $\mathrm{Bu}_{4} \mathrm{SbBr}$ coencapsulated with various aromatic neutral species, ${ }^{74}$ or cobaltocenium and ferrocenium cations that show high affinity, the latter allowing reversible assembly and deassembly of the capsule driven by electrochemical stimulus. ${ }^{75}$ The large cavity is roomy enough to host an entire host-guest complex composed of a calix[4]arene and tetramethylammonium cation in a kind of nanometric Russian doll. ${ }^{76}$ Some neutral polar guests showed affinity for the hexameric capsule, in particular, using diffusionordered NMR spectroscopy it was eventually demonstrated that six molecules of glutaric $\mathrm{acid}^{77}$ or three molecules of $\beta$-methyl-D-glucopyranoside are hosted by the supramolecular aggregate, ${ }^{78}$ conversely to what was assumed at the end of $1980 \mathrm{~s}$ when $1: 1$ host-guest interactions were described. 
Figure 14 A large hexameric capsule that can accommodate

Pyrogallo[4]arene is a very similar structure com to resorcin[4]arene bearing an extra phenol residu inbetween the other two. Such subunits self-assemble, to roing hexameric assemblies, but here the presen of $/$ vater is not necessarily required ${ }^{79}$ as extra hydrogn ondir $f$ residues are intrinsically present in the mo a er ng the hexamer with 72 hydrogen bonds. The se - rembly behavior is dependent on the synthetic pro are adopted, in particular, crystallization from ane methanol with little amounts of nitrobenzen or 0 dorobenzene, and subsequent dissolution in even ar organic solvents ensures the formation of the he cum cale. ${ }^{80}$ The large stability imparted by the nur erous hy rogen bonds maintains the hexameric structure fo the psule even in $50 \%$ $\mathrm{v} / \mathrm{v}$ mixture of acetone- $d$ and $\mathrm{D}_{2} \mathrm{O}$. rypical apolar organic solvents are chloroform $l$ or ben ene- $d_{6}$ and, in these cases, the capsule contains se ral molecules of solvent characterized by different ro nances probably because slightly different enviror in the cavity. ${ }^{81}$

On mixingequal amounts of pyrogallo[4]arene and resorcin[4]arene was foune as a con equence of the extremely high selfrecognition press hat caused the formation of only capsules constructed from the same macrocycle. Guest affinity is also different between the two capsules, with pyrogallo[4]arene that do not provide binding for alkylammonium cations but prefer encapsulation of tertiary amines ${ }^{82}$ or alkanes. ${ }^{83}$ Large aromatic polycyclic molecules such as
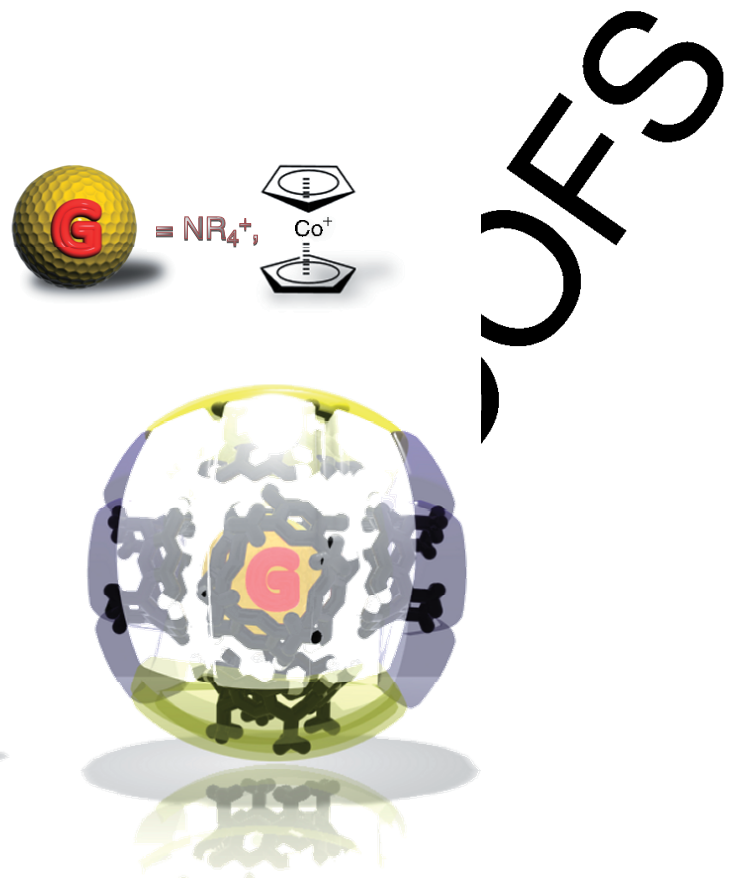

solven folecules or cationic guests.

pyrene butyric acid (PBA) are suitable guests as evidenced in solution by means of spectrophotometric studies. PBA was employed as a fluorescence probe showing double coencapsulation within the hexameric capsule with the two guests kept well separated owing to the specific interactions with the capsule walls. 84

\section{HYDROGEN-BONDING HETEROMERIC CAPSULES}

\subsection{Dimeric capsules}

\subsubsection{Tetraacid-resorcin/4/arene with tetrapyridil-resorcin[4]arene}

A hydrogen bond in noncompetitive solvents between a carboxylic acid and pyridine is a common synthon for the construction of supramolecular aggregates. The presence of cavitands based on the resorcin[4]arene scaffold rigidified by methylene connections between adjacent oxygen atoms, one endowed with four $\mathrm{COOH}$ and one with four 3-pyridil moieties form unidentified aggregates in chloroform- $d$ but give rise to clear heterodimeric capsules if a solvent of correct volume and shape to be encapsulated is provided, like $p$-xylene- $d_{10}$ or $\mathrm{CDCl}_{2} \mathrm{CDCl}_{2}$, once again confirming the principle that cavities cannot remain empty but require the presence of molecules 

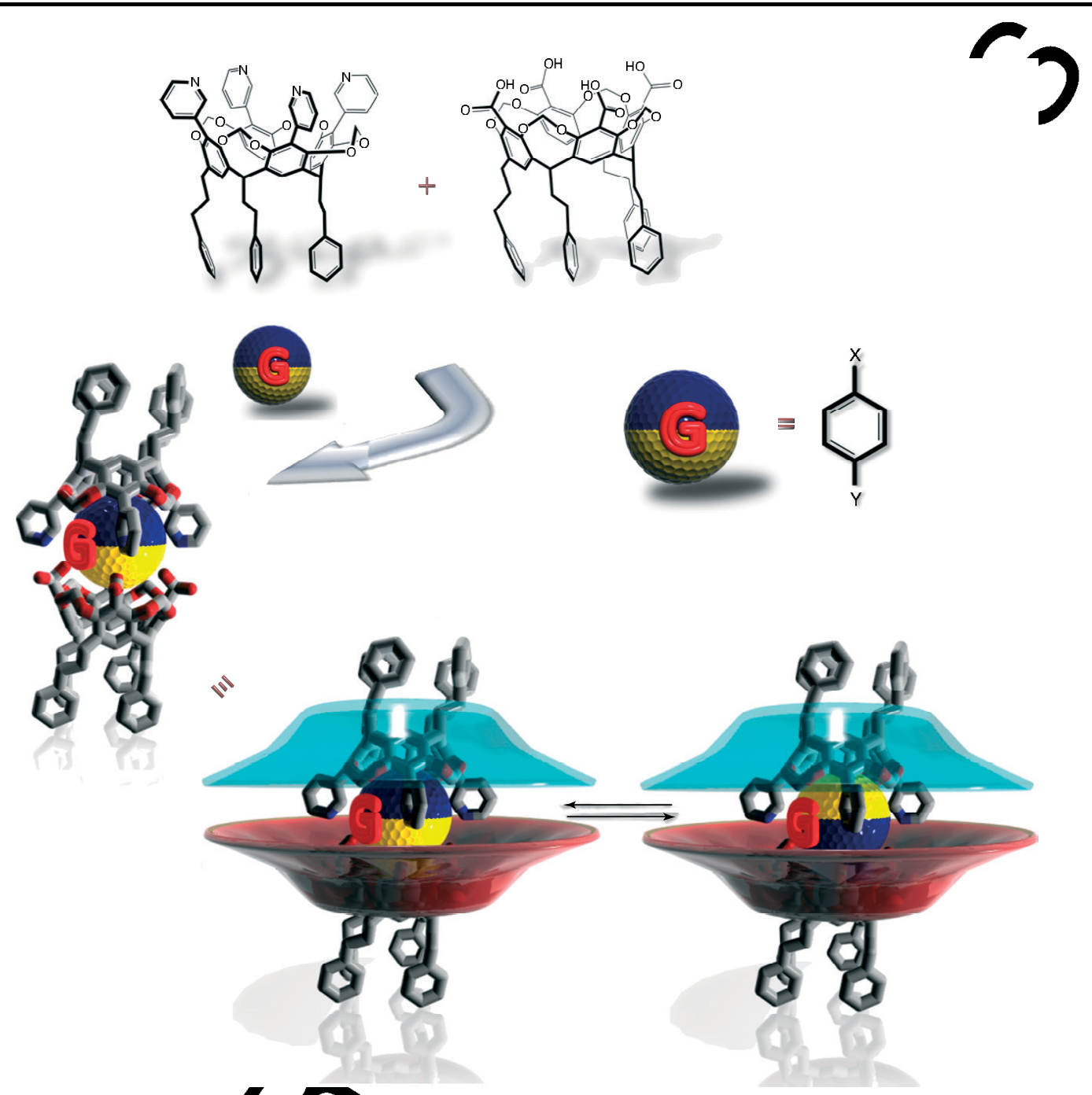

Figure 15 Hydrogen-bonded heterod ric capsule featuring orientational isomerism for encapsulated guests

for salvation of internal turaces Elongated molecules like $p$-disubstituted bo ache syoums have the right shape and average volum to fill a cavity of this kind of dimeric heterocansul with increased capsule formation in the order $p$-et ltourne $<1$-ethyl-4-iodobenzene $<$ 1-iodo-4-meth $>1,4$-dimethoxybenzene $<1,4-$ diiodobenzere, sogesting that $\mathrm{CH}-$ halogen and $\mathrm{CH}-\pi$ interactions betren the guest with the methylene groups of the sorcinar he play a pivotal role in the guestinduced as abl of the capsule. ${ }^{85}$ In particular, guests with different substituents in position 1 and 4, when encapsulated, provide two possible isomeric assemblies as a function of the orientation of the substituents of the guest either toward the tetracarboxylic or toward the tetra-3pyridil pole of the capsule as the resulting balance of weak attractive and repulsive interactions of the groups with the two halves of the capsule. Extensive investigation with a series of different substrates allowed to demonstrate the preferential allocation of iodo and methoxy groups preferentially oriented toward the pyridyl pole in the first one and toward the carboxyl pole in the methoxy group (Figure 15). ${ }^{86}$

A smaller capsule with a semispherical shape based on the same combination of hydrogen bonds was obtained using the tetracarboxylic cavitand paired with meso-tetra(2pyridyl)porphyrin with a rather small cavity suitable for encapsulation of gases and small molecules as guests. ${ }^{87}$ The binding affinity for gases was in the order $\mathrm{CH}_{4}<$ $\mathrm{HC} \equiv \mathrm{CH}<\mathrm{H}_{2} \mathrm{C}=\mathrm{CH}_{2}$ in 1,1,2,2-tetrachloroethane- $d_{2}$ as a noncompetitive solvent with separate signals for bound and 

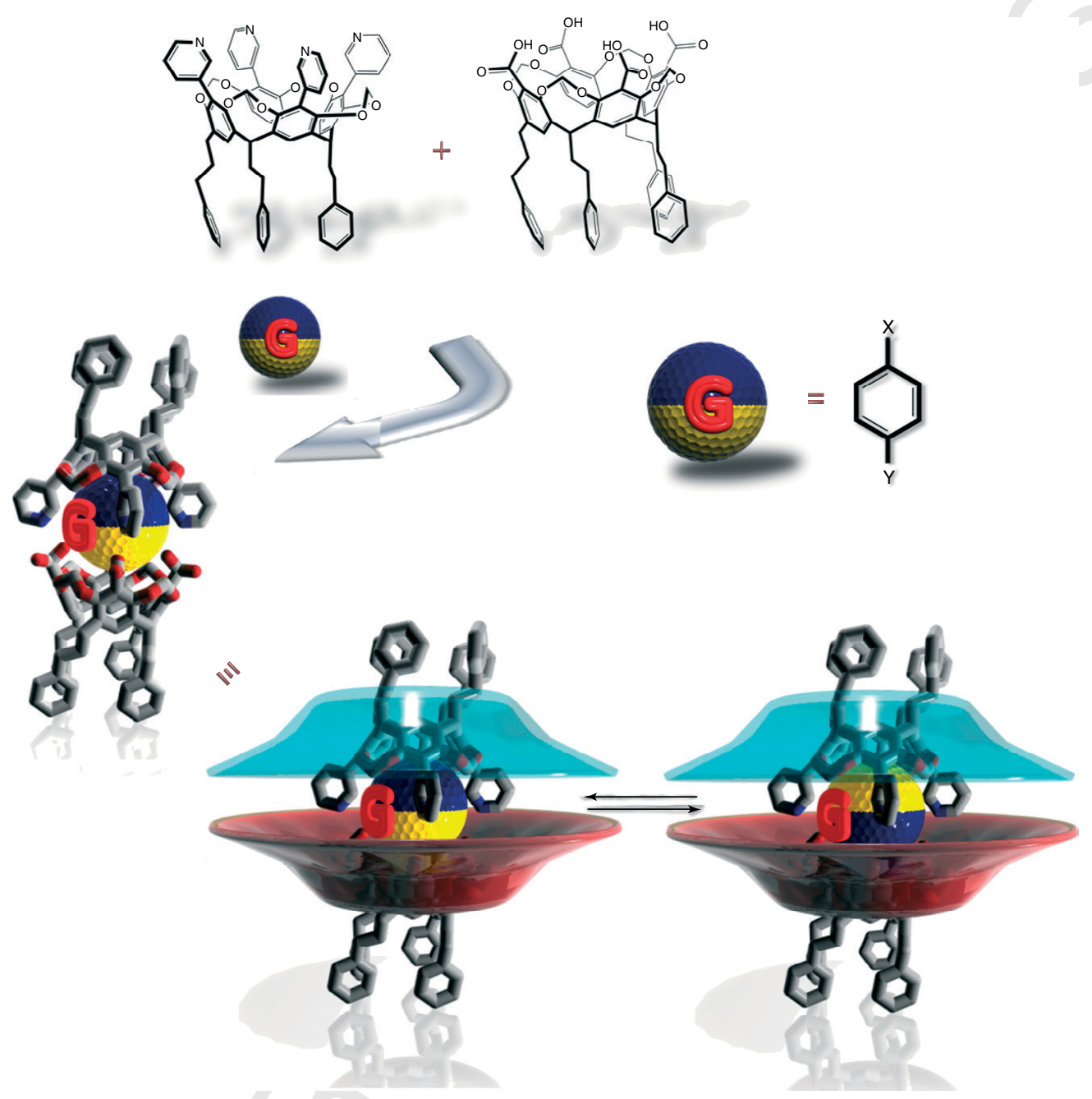

Figure 15 Hydrogen-bonded heterodimeric capsule featuring orientational isomerism for encapsulated guests.

for salvation of internal surfaces. Elongated molecules like $p$-disubstituted benzene systems have the right shape and average volume to fill a cavity of this kind of dimeric heterocapsules, with increased capsule formation in the order $p$-ethyltoluene $<1$-ethyl-4-iodobenzene $<$ 1 -iodo-4-methoxybenzene $<1$,4-dimethoxybenzene $<1,4$ diiodobenzene, suggesting that $\mathrm{CH}-$ halogen and $\mathrm{CH}-\pi$ interactions between the guest with the methylene groups of the resorcinarene play a pivotal role in the guestinduced assembly of the capsule. ${ }^{85}$ In particular, guests with different substituents in position 1 and 4, when encapsulated, provide two possible isomeric assemblies as a function of the orientation of the substituents of the guest either toward the tetracarboxylic or toward the tetra-3pyridil pole of the capsule as the resulting balance of weak attractive and repulsive interactions of the groups with the two halves of the capsule. Extensive investigation with a series of different substrates allowed to demonstrate the preferential allocation of iodo and methoxy groups preferentially oriented toward the pyridyl pole in the first one and toward the carboxyl pole in the methoxy group (Figure 15)..$^{86}$

A smaller capsule with a semispherical shape based on the same combination of hydrogen bonds was obtained using the tetracarboxylic cavitand paired with meso-tetra(2pyridyl)porphyrin with a rather small cavity suitable for encapsulation of gases and small molecules as guests. ${ }^{87}$ The binding affinity for gases was in the order $\mathrm{CH}_{4}<$ $\mathrm{HC} \equiv \mathrm{CH}<\mathrm{H}_{2} \mathrm{C}=\mathrm{CH}_{2}$ in 1,1,2,2-tetrachloroethane- $d_{2}$ as a noncompetitive solvent with separate signals for bound and 
free gaseous guest in solution even at room temperature as the slowest guest exchange rate so far reported for gaseous guests in self-assembled hosts.

\subsubsection{Tetraphenol-resorcin[4]arene with tetrapyridil-resorcin[4]arene}

Phenol groups are less acidic compared to carboxylic groups but with pyridine they provide sufficiently strong hydrogen bonds. The research group of Kobayashi investigated the reversible self-assembly of hetero capsules between tetrakis(4-hydroxyphenyl) cavitand and tetra(4-pyridyl)-cavitand with resorcin[4]arene scaffold (Figure 16) ${ }^{88}$ As observed for the above-mentioned capsule from the same research group, $p$-disubstituted aromatic or 2,6-disubstituted naphthyl substrates enable sufficient assembly of the ternary structure composed of the dimeric capsule and the guest. An extensive study of a wide range of substrates ${ }^{89}$ demonstrated that the capsule discriminates guests on the basis of sizes that should be in the range
12.1-15.2 $\AA$ with strong differences even for one extra carbon atom; deuterated guests were worse compared to their protio counterparts because of the higher polarizability of $\mathrm{C}-\mathrm{H}$ compared to that of $\mathrm{C}-\mathrm{D}$ that favors $\mathrm{CH}-\pi$ contacts with the aromatic concave surfaces of the cavity. Orientational isomerism of the hetero capsule with the bound guest confirmed the preference of halogen substituents for the electron-poor tetrapyridin-cavitand pole owing to favorable halogen $-\mathrm{CH}$ and halogen $-\pi$ attractive interactions derived by the polarization of the I atom that presents $\delta+$ character in the polar region and $\delta-$ in the equatorial area of the $\mathrm{C}-\mathrm{I}$ bond.

1,4-Diacetoxybenzene derivatives are another class of good guests that show restricted tumbling inside the capsule owing to their particular size, shape, and attractive $\mathrm{CH}-\pi$ contacts with the internal aromatic surfaces. If such guests are substituted in positions 2,5 with alkoxy residues, the spinning rate for rotation of the guest along the vertical axis decreases with increase in the length of
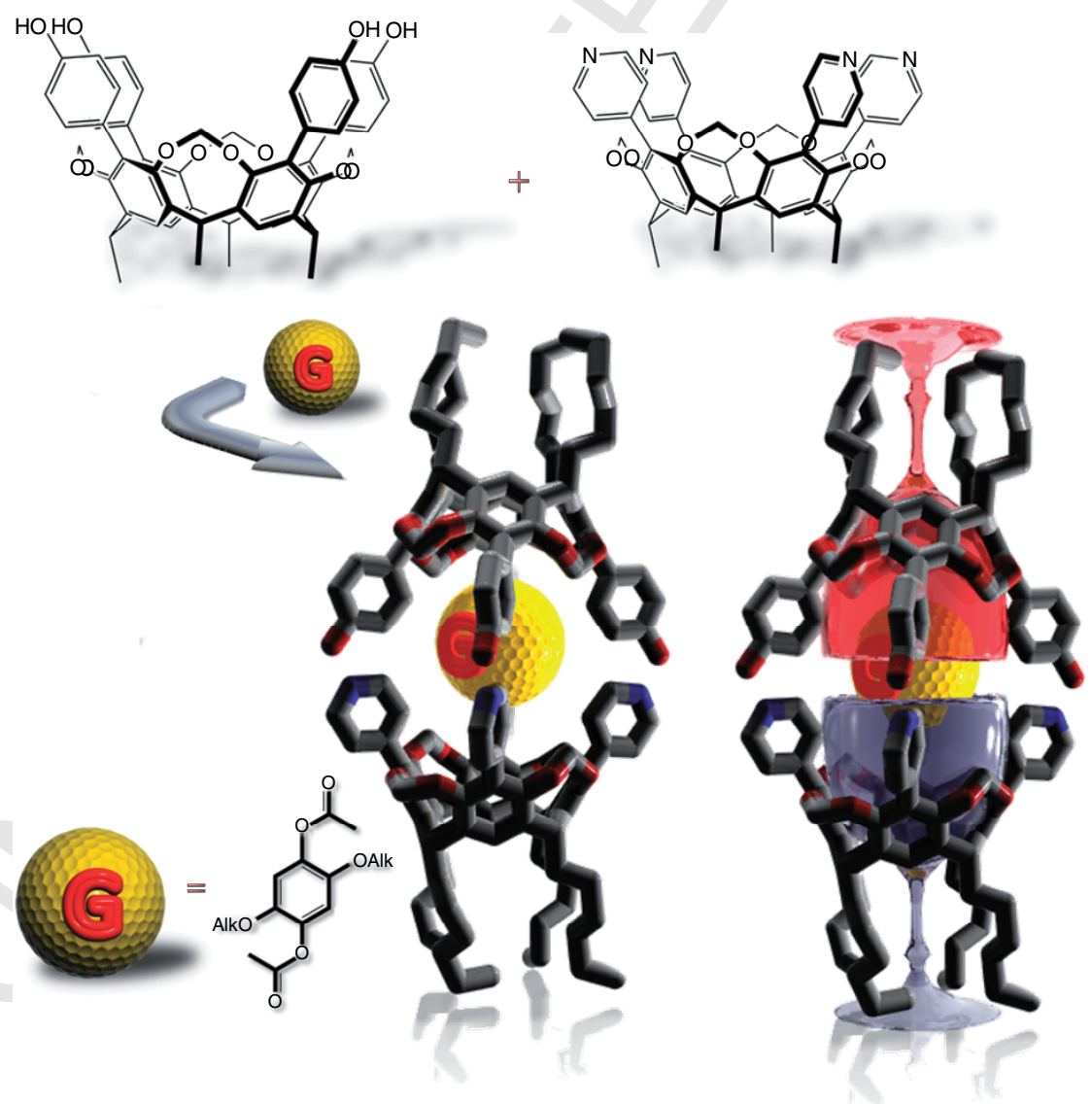

Figure 16 Hydrogen-bonded heterodimeric capsule featuring slow spinning for encapsulated tetrasubstituted aromatic guests. 
the alkoxy group because of increased frictions with the capsule. The guests resemble the rotator that spins in the capsule as stator of the self-assembled supramolecular gyroscope. ${ }^{90}$

\subsubsection{Tetraimide-resorcin[4]arene cavitand and resorcin[4]arene}

In the former capsules, the formation of heterodimers is driven by the presence of a strong hydrogen bond donor in one cavitand and a strong hydrogen bond acceptor like pyridine residues in the other, thus making the homodimerization process highly disfavored. Rebek and collaborators demonstrated that formation of hetero dimeric capsules can be driven by the size and shape of the guest as in the case of resorcinarene monomer and tetraimide-cavitand. The two units in chloroform- $d$ give rise to three capsule, the hexameric resorcinarene filled with six molecules of solvent, the dimeric cylindrical capsule with three chloroform guests, and a new hybrid capsule ${ }^{91}$ (Figure 17) containing two molecules of chloroform derived by hydrogen bonding between resorcinarene and tetraimide cavitand. The amount of the hybrid capsule is dependent on the guest. In fact, in mesitylene- $d_{12}$ as a noncompetitive solvent, midsize guests, such as 1,4-diethylbenzene or $p$-cyclophane that are not singularly encapsulated either in the hexamer or in the cylindrical capsule, drive the exclusive formation of the new heterocapsule where orientational stereoisomerism is observed with $p$-cymene depending on the position of the methyl and isopropyl group with respect to the deep or shallow pole of the capsule.

\subsubsection{Tetraimide-resorcin[4]arene cavitand and monomer of tennis ball}

A second different hybrid capsule was obtained by mixing tetraimide-cavitand with the grycoluril unit of the well-known tennis ball with complete disappearance of the dimeric homocapsules indicating that in chloroform- $d$ the hybrid dimmer is preferred owing to the good match between the imido hydrogen bond donor and ureido carbonyls as good hydrogen bond acceptors (Figure 18)..$^{92}$ Suitable guests for the present hybrid capsule are the same as the previous one, in particular, 2,2- $p$-cyclophane snug fit in the cavity giving rise to slow rotation along the vertical axis on the NMR timescale with an activation barrier of about $15.3 \mathrm{kcal} \mathrm{mol}^{-1}$ between the two enantiomeric complexes. Other elongated guests such as 2-methyl heptane, 2,2-dimethylhexane, $p$-ethyltoluene, and $p$-cymene are easily encapsulated but are too long to freely tumble in the cavity showing two orientations in the capsule in slow exchange with increasing preference to allocate the small end toward the tapered end of the cavitand pole for strong $\mathrm{CH}-\pi$ contacts.


Figure 17 Heterodimeric capsule between a cavitand and resorcin[4]arene templated by the presence of suitable guests. 


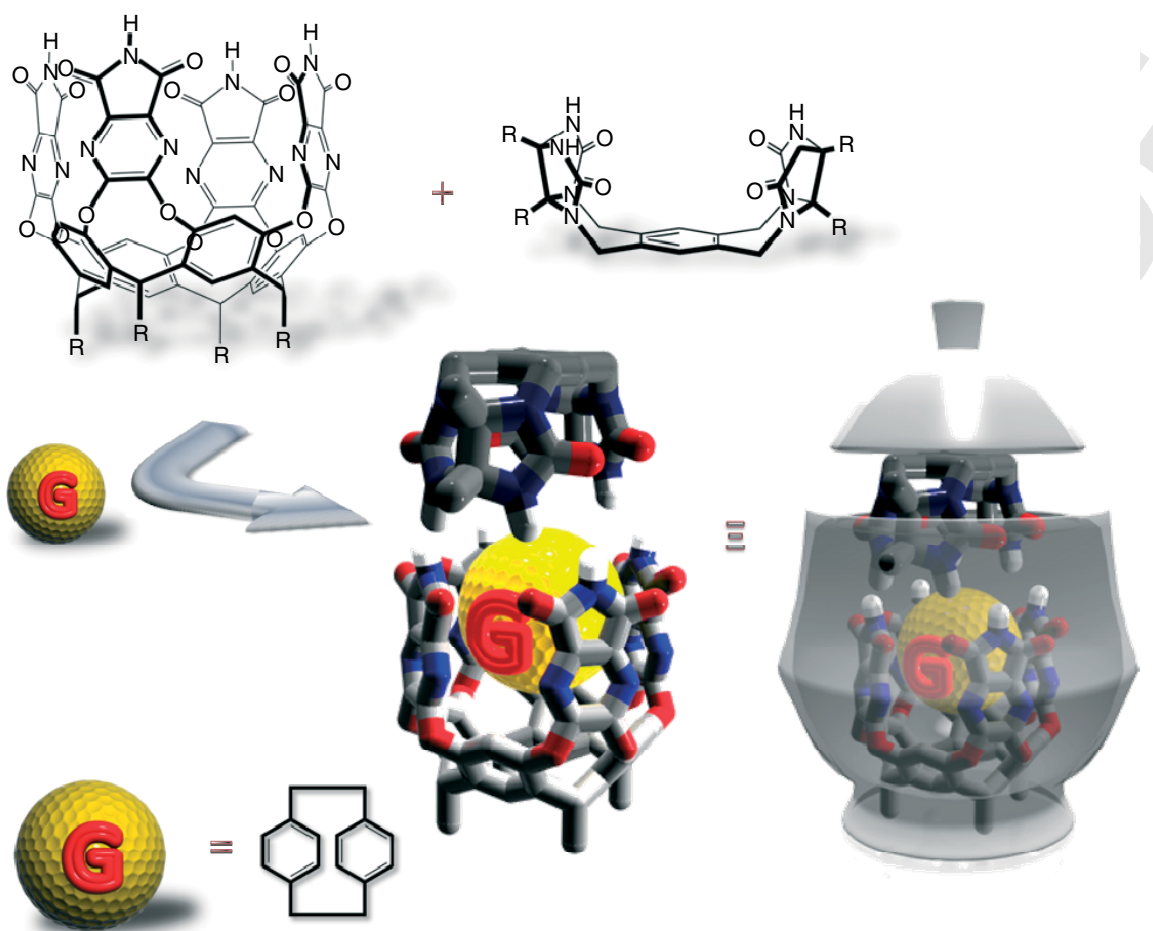

Figure 18 Heterodimeric capsule between a cavitand and a tennis ball monomer templated by the presence of suitable guests.

\subsection{Multimeric capsules}

\subsubsection{Tetraacid-resorcin[4]arene with 2-amino-pyridine or tetrahydro-2- pyrimidinone}

Large capsules are possible via interaction of several units using small organic molecules bearing functional groups that are able to both provide hydrogen bond donors and acceptors like 2-aminopyridine $(2-\mathrm{AP})^{93,94}$ or tetrahydro-2-pyrimidinone (THP). ${ }^{94}$ Overall six components self-assemble into an elongated capsule through the formation of 16 hydrogen bonds between two cavitands held together in a rim-to-rim fashion involving four molecules of 2-AP or THP. The structure was confirmed in solution by NMR analysis in chloroform$d$ and encapsulation of medium-sized guests like 2,6-dimethoxynaphthalene and 2,6-dibromonaphthalene, and $p$-diiodobenzene was possible when using a shorter tetracarboxyl-resorcin[4]arene, while very large guests like hexakis(4-methoxyphenyl)benzene or hexakis(4iodophenyl)benzene were encapsulated using an extended tetracarboxyl-resorcin[4]arene as illustrated in Figure 19. In particular, guest selectivity was fine-tuned using the same tetraanionic cavitand as that with 2-AP, which led to the encapsulation of hexakis(4-iodophenyl)benzene, while with THP the capsule selectively hosted hexakis(4methoxyphenyl)benzene.

\subsubsection{Tetraimide-resorcin[4]arene with glycoluril}

Adequate matching of different subunits to form hybrid capsules depends strongly on the templating effect of the guest. Its size and shape can be used to select the one that provides the best cavity among several possible stoichiometry. As observed before, tetraimide cavitand and glycoluril not only form dimeric heterocapsules with midsize guests but also enable the formation of new, even larger capsules when using long alkanes as templating guests. A dimeric cylindrical capsule was described to bind tetradecane in a helical conformation but pentadecane was too long to fit the cavity. Addition of excess of glycoluril to the tetraimide cavitand in the presence of alkanes from $\mathrm{C}_{15} \mathrm{H}_{32}$ to $\mathrm{C}_{19} \mathrm{H}_{40}$ led to the formation of new extended cylindrical capsules with increased length of $\sim 7 \AA$ and volume of $\sim 200 \AA^{3}$ (Figure 20) where four molecules of glycoluril intercalate in the polar belt of the imide moieties. ${ }^{95}$ The new capsule can adopt two enantiomeric arrangements of the intercalating glycoluril residues, and methylene residues of the alkyl guests become diastereotopic in the nanometric 

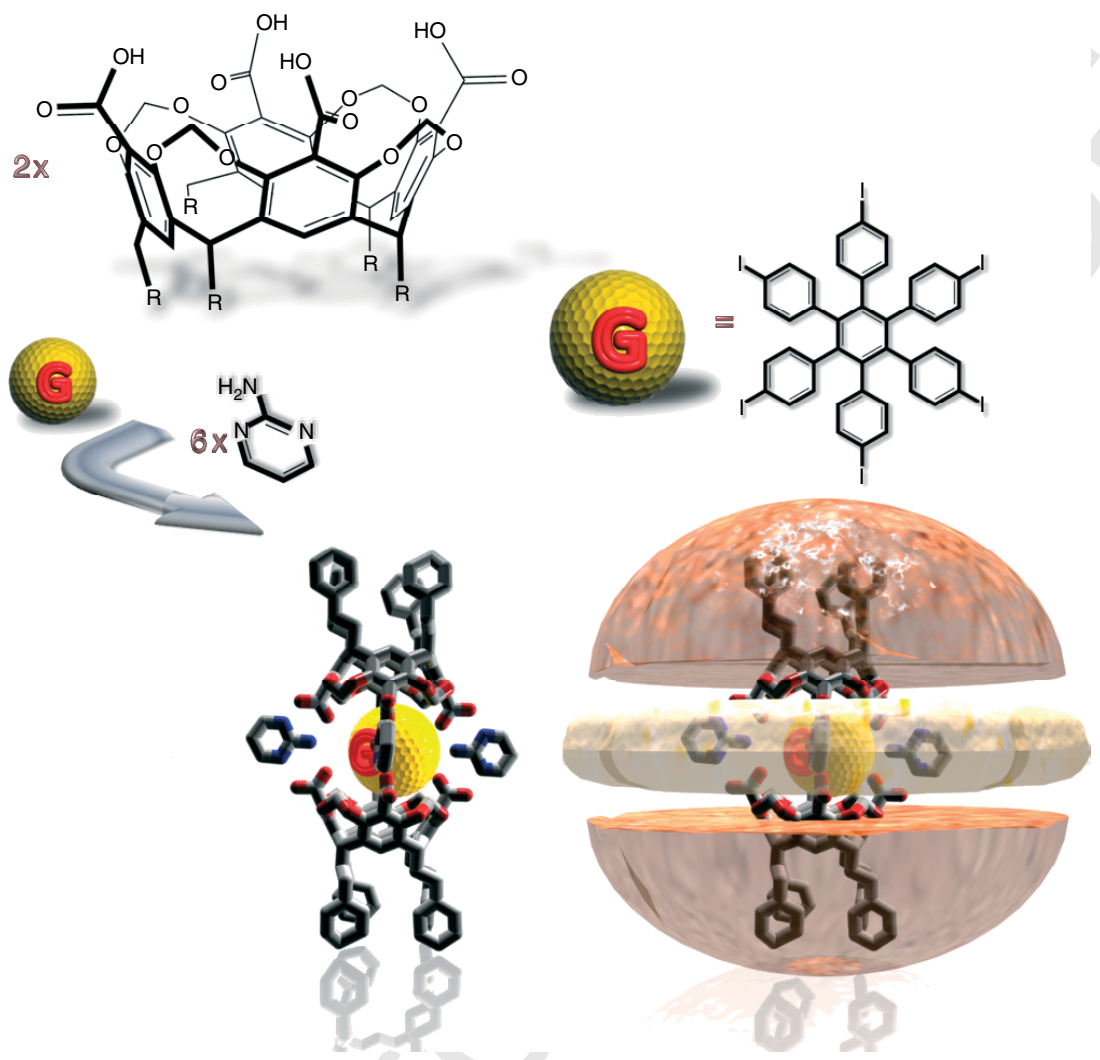

Figure 19 Multimeric hydrogen-bonded capsule showing encapsulation of large flat aromatic guests.

chiral environment. Interconversion of the two enantiomeric extended capsules is dependent on the size and coiling of the guest: in fact, the more the guest fills the cavity and the more it exerts pressure on the glycoluril belt, the more it favors interconversion of the enantiomers that occur at higher rates. ${ }^{96}$ Using glycoluril endowed with amino residues in the side chains, it was possible to prepare a reversible spring-loaded device where tetradecane is encapsulated in its extended conformation and, upon addition of $\mathrm{HCl}$, which protonates the amino groups and causes precipitation of the glycoluril, the capsule become shorter and the guest is forced to assume a helical conformation. ${ }^{97}$ Even longer capsules, hyper-extended self-assembled containers, with two and three belts each composed of four glycoluril molecules can self-assemble, reaching the maximum internal length of $35 \AA$ in the presence of alkanes up to $\mathrm{C}_{26} \mathrm{H}_{54}$ basically driven by optimization of $\mathrm{CH}-\pi$ contacts between the guest and the inner surfaces of the capsule that cannot efficiently be solvated by the bulky mesitylene$d_{12}$ used as solvent. ${ }^{98}$ Extended capsules with one belt of glycoluril can accommodate more than one guest like two molecules of $p$-xylene or four molecules of cyclopropane, ${ }^{99}$ and even xenon coencapsulated with a longer guest such as nonane through dodecane. ${ }^{100}$ When 1,4-disubstituted aromatic guests such as $p$-ethyltoluene were studied, three isomeric capsules were present, depending on the reciprocal orientation of the social isomers in the capsule that could be with both methyl groups in the middle (28\%), both ethyl groups in the middle $(68 \%)$, and only $4 \%$ of head-tail isomer even though the latter is statistically the more favorable but overall permits worse $\mathrm{CH}-\pi$ contacts with the walls of the cavity. ${ }^{101}$

\subsubsection{Rosettes}

Rosettes are beautiful supramolecular aggregates that form spontaneously when three calix[4]arene dimelamines units and six barbiturates or cyanurate are mixed in a $1: 2$ ratio in apolar solvents. ${ }^{102}$ These assemblies, made of nine achiral components, are held together by 36 hydrogen bonds that provide the driving force for the assembly, and can adopt up to three diasteroisomeric forms with $D_{3}$ (chiral), $C_{3 \mathrm{~h}}$, and $C_{\mathrm{s}}$ symmetry due to the reciprocal staggering or aligning of the dimelamine units. Rosettes have been thoroughly studied for their unique features 


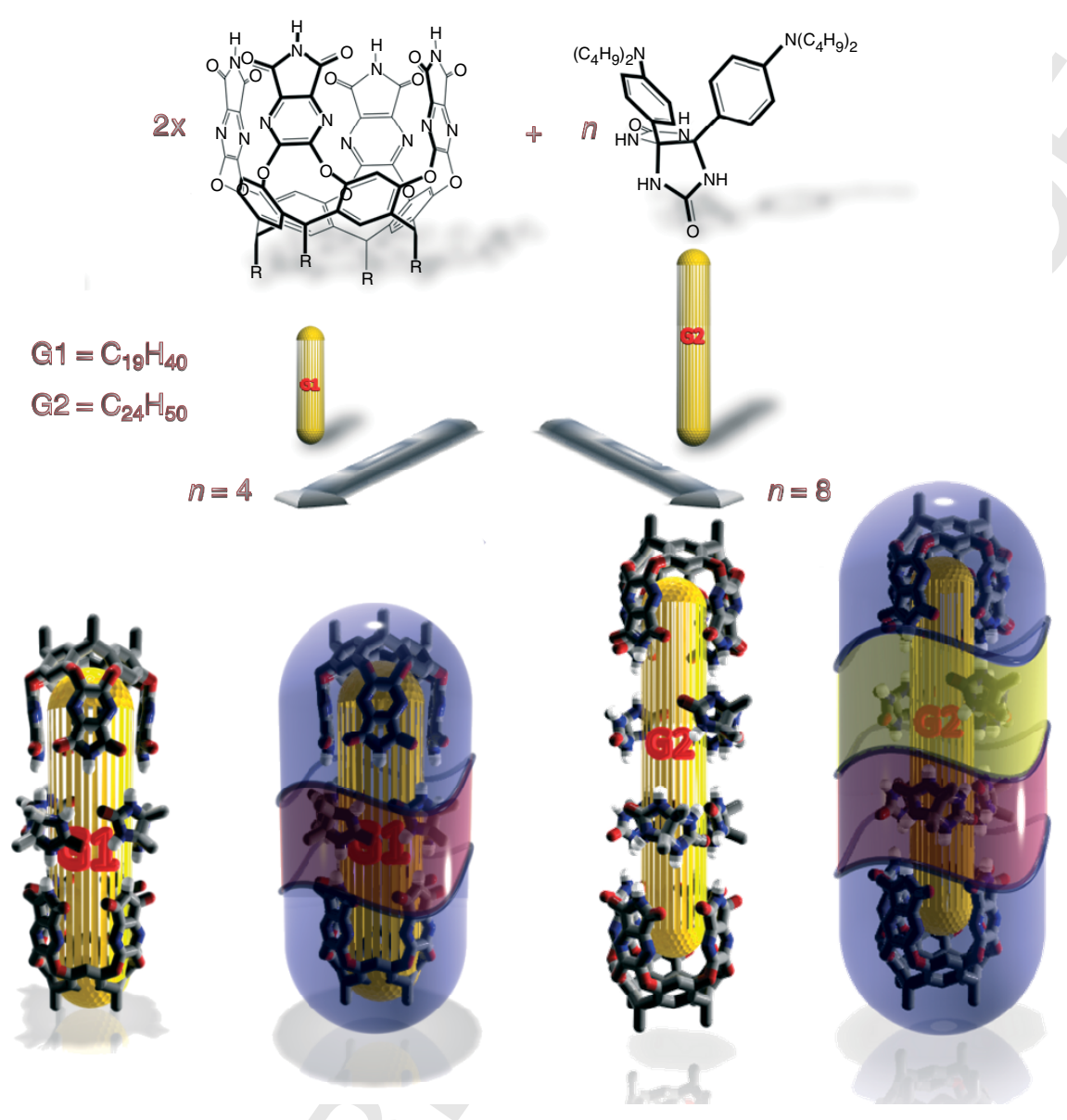

Figure 20 Guest templated formation of elongated cylindrical capsules between cavitand and glycoluril monomer.

in terms of supramolecular chirality and chiral memory effect, ${ }^{9}$ but intriguing encapsulation phenomena are also possible with these heteromeric capsules characterized by a disclike cavity. Anthraquinone derivatives ${ }^{103}$ are electronrich flat guests that, if adorned with hydroxy groups in certain positions like for alizarin, can be encapsulated in the rosettes forming a network of interguest hydrogen bonds and embarking in host-guest attractive $\pi-\pi$ contacts (Figure 21). ${ }^{104}$ Binding of this class of guests to rosettes is favorable, with $K_{\mathrm{a}} \sim 10^{10} \mathrm{M}^{-3}$ observing a remarkable adaptation of the host, which switches symmetry from staggered to eclipsed and increases the distance between the floors of the assembly to fit the triplet of guests as a good example of a guest-induced fit.

The system has been further elaborated preparing rosettes bearing pyridyl residues attached to the external periphery of the assembly ${ }^{105}$ and able to make hydrogen bonding with dicarboxylic acids. ${ }^{106}$ This class of rosettes enabled exclusive endo binding of anthraquinone guests allocated within the cavity and exo binding of up to three dibenzoyl-D-tartaric acid molecules on the outer of the capsule.

\section{ION-PAIRING HETEROMERIC CAPSULES}

Ionic interactions between positively and negatively charged residues have been much less investigated compared to hydrogen bonds and have rarely been implemented into supramolecular assemblies. In fact, due to solubility concerns, the construction of supramolecular aggregates held together by ionic couples is a more challenging task because often charge pairing could cause a net decrease in solubility of the aggregate, resulting in precipitation. Because of the difficulty in mastering ionic interactions that are less directional compared to hydrogen bonding, rare examples of multimeric capsules are known; basically, 


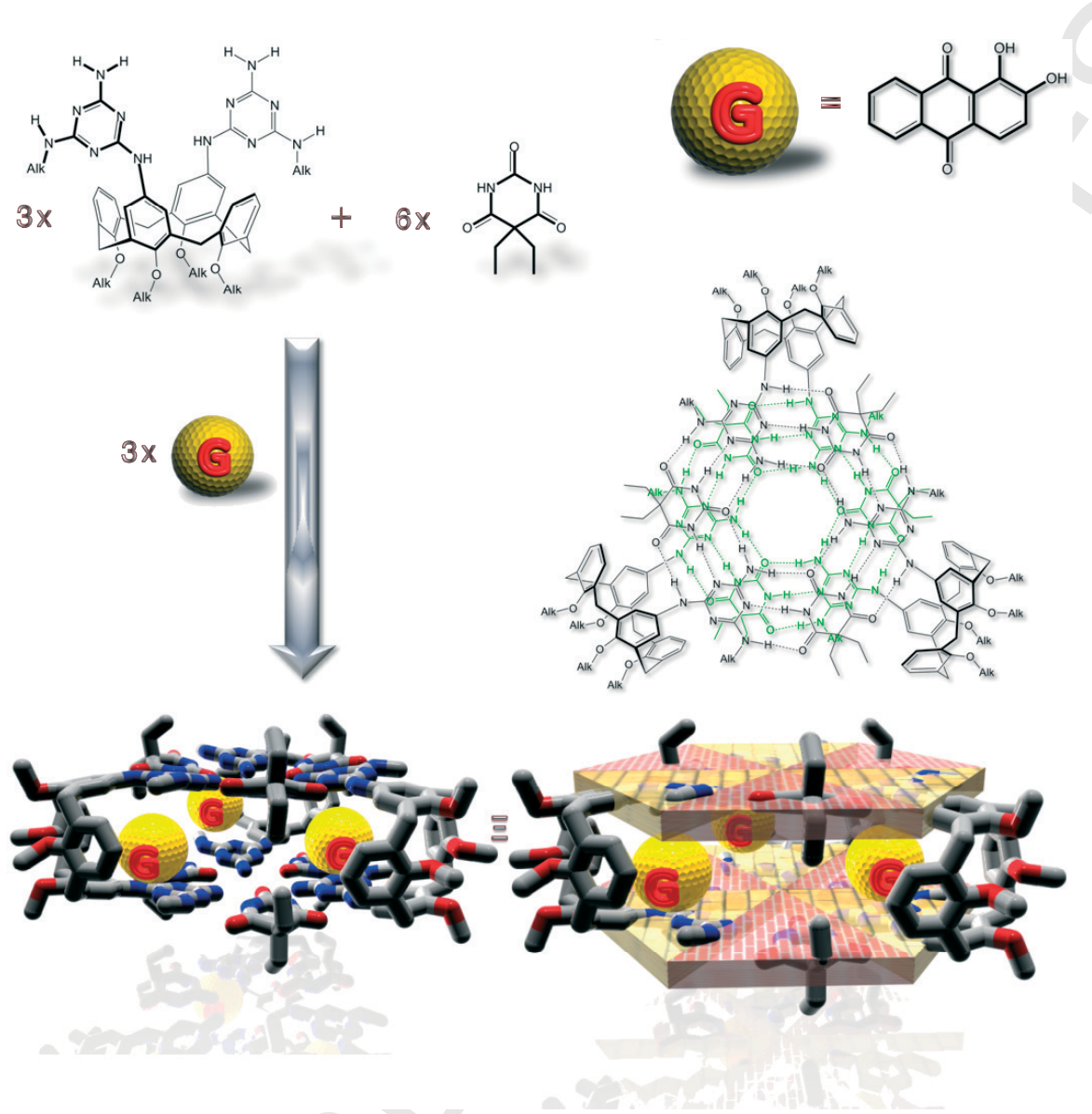

Figure 21 Encapsulation of triplets of alizarin neutral guests within the cavity of self-assembled rosettes.

only heterodimeric capsules where one subunit is positively and the other negatively charged.

\subsection{Dimeric capsules}

The construction of self-assembled capsules based on ionic interactions to be tested as supramolecular hosts in polar solvents such as alcohols or water is a timely goal that, compared to hydrogen-bonded capsules, is still in its infancy even though important steps have been taken. Few examples have been reported regarding ionic capsules between cationic flat tetrapyridinium-Zn(II)-porphyrinate and anionic tetrasulfonate-calix[4]arenes ${ }^{107}$ that form a hemispherical entropy-driven capsule that displayed high stability in the presence of water or competing salts. Apart from solvent molecules, such capsules showed encapsulation of small guests like 1-methylimidazole or 4-methylpyridine via coordination to the $\mathrm{Zn}$ (II) metal center, while bulkier caffeine was bound outside on the opposite side of the porphyrin plane. ${ }^{108}$
All the other dimeric ionic capsules are based on two oppositely charged calix[4]arene scaffolds that dimerize in solution owing to the presence of one subunit of typical hydrophilic anionic groups such as sulfonate, phosphonate, and carboxylate that make ion pairing with cationic groups such as ammonium and anilinium present in the second subunit. The binding affinity is, as expected, dependent on the $\mathrm{p} K_{\mathrm{a}}$ of the functional groups involved, with stronger associations observed for dimers characterized by larger differences in $\mathrm{p} K_{\mathrm{a}}$ between anionic and cationic counterparts, with values on the order $10^{4}-10^{7} \mathrm{M}^{-1}$, for example, tetrabenzylphosphonate-calix[4]arene prefers tetraammonium-calix[4]arene compared to tetraaniliniumcalix[4] arene for about 2 order of magnitude. ${ }^{109,110}$ In most of the cases, thermodynamic investigation showed that the association process in methanol-water media is strongly entropy driven, most likely due to the release of highly ordered solvent molecules in the bulk solvent. ${ }^{111}$ Weaker interactions are therefore present for dimeric capsules based on ammonium and carboxylate functional groups 


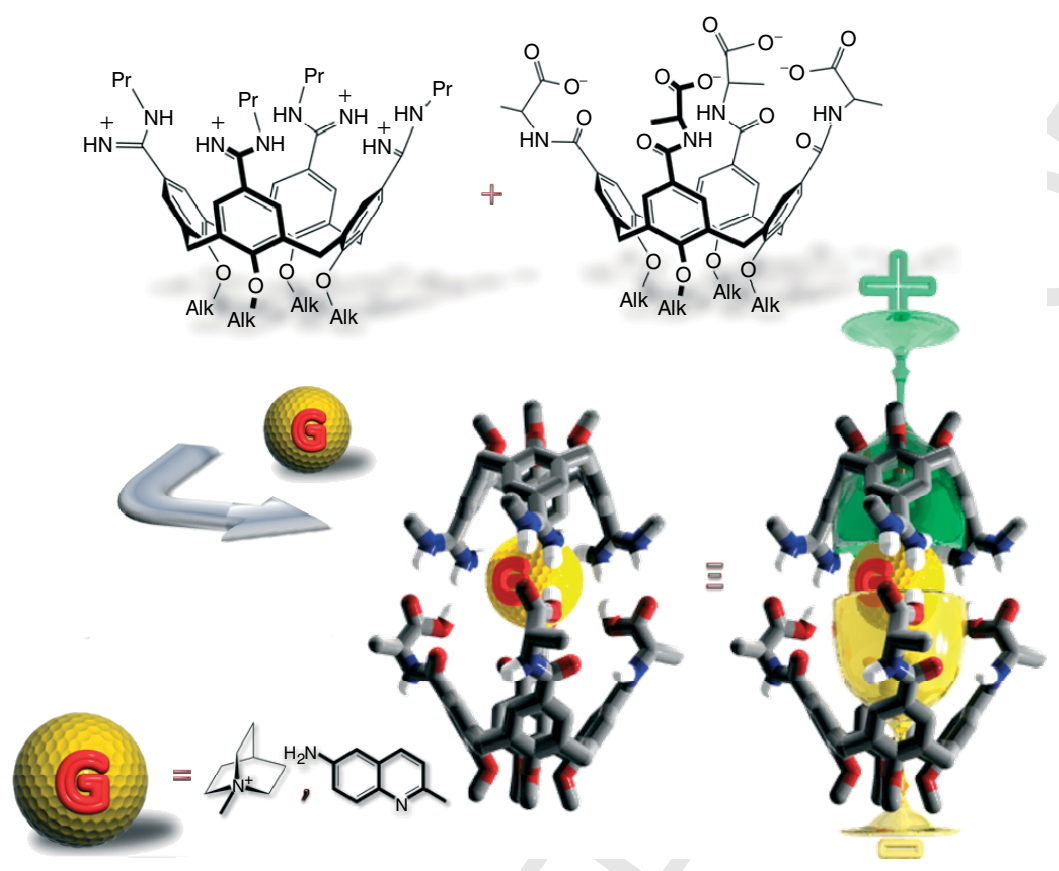

Figure 22 Ionic heterodimeric capsule based on tetraalkylamidinium-calix[4]arene with a tetracarboxylate-calix[4]arene as host for cationic and neutral guests.

that provide calix[4]arene-based capsules with association constants of about $10^{3} \mathrm{M}^{-1}$ in dmso- $d_{6}$ with $5 \%$ of buffer at $\mathrm{pH}$ 6.5. ${ }^{112}$ None of the above-mentioned ionic capsules displayed clear-cut examples of guest binding, albeit it is likely that the cavity of such capsules is occupied by solvent.

\subsubsection{Tetraamidinium-calix[4]arene and tetraanionic-calix[4]arenes}

A step forward was accomplished using tetraalkylamidinium-calix[4]arenes as cationic subunits that showed easy dimerization with tetrasulfonate-calix[4]arene ${ }^{113}$ under fast exchange on the NMR timescale. Dimerization was proved via NMR analysis that showed an upfield shift for the protons of the propyl amidinium chains compared to the simple tetracationic scaffold in solution, as an indication of dimer formation via inclusion of the alkyl chain inside the cavity. Upon addition of the guest, the alkyl chains are displaced by the incoming molecule indicating that a certain degree of hydrophobic effect contributes to the binding ability of the guests in the apolar and weekly solvated core of the capsule. A suitable guest was $N$-methylquinuclidinium and also tetramethylammonium or acetylcoline with good association constants in the range $20-170 \mathrm{M}^{-1}$.

Pairing of the tetraalkylamidinium-calix[4]arene with a calix[4]arene scaffold bearing $N$-coupled L-alanine carboxylate anionic groups on the wider rim led to the formation of another heterodimeric ionic capsule (Figure 22) ${ }^{114}$ that not only showed similar encapsulation behavior compared to the former one toward cationic guests but also enabled encapsulation of neutral guests. A wide range of possible candidates was computationally investigated concluding that 6-amino-2-methylquinoline was a good guest with a binding constant for the capsule of $2 \times 10^{3} \mathrm{M}^{-1} .115$ The presence of the L-alanine residues imparts chirality to the capsular assembly but thus far examples of enantioselective binding have not been observed, even though the ionic interactions that hold together the capsule provide an association constant as high as $10^{5} \mathrm{M}^{-1}$ in pure water.

\subsection{Multimeric capsules}

Multimeric capsules are rare; one example in water is based on ion pairing between two tris(imidazoline) scaffold with two tartaric acid, leading to a capsular assembly with no cavity. In this system, proton exchange between the acid and the base provide strong multiply charged interactions with hydrogen bond contacts strong enough to enable aggregation in pure water with $\mathrm{p} K_{\mathrm{a}} \sim 6$. $^{116}$

A more complex multimeric ionic capsule has been reported few years ago and is based on the interaction between two tetracationic molecules of tetrakis(pyridiniummethyl)teramethyl cavitands that do not reject as expected but face each other owing to the insertion 
of four anionic species between two different pyridyl moieties thus forming a capsular structure in methanol- $d_{4}$ with a cavity where one or two anions, depending their size, can be accommodated. ${ }^{117}$ The driving force for the aggregation comes form the formation of four pyridinium-anion-pyridinium attractive interactions, and this capsule exists in equilibrium with the analogous comprising only three triple-ion interactions. The latter aggregate presents one available binding site formed by two relatively closed pyridinium moieties that can form pyridinium-guest-pyridinium interactions with neutral electron-rich aromatic guests like $p$-iodophenol or $p$-iodoaniline. In the latter case, interaction of the guest does not occur within the cavity of the capsule but binding takes place on the equatorial area of the capsule.

\section{HYDROPHOBIC CAPSULES}

While formation of capsular self-assembled systems based on hydrogen bonding or ion pairing can be, in some way, predicted on the basis of the distribution of functional groups, shape, and the form of subunits, the same is not valid and serendipity takes the lead when the self-assembly process is driven by the hydrophobic effect. The latter, in its more common version, is based on the entropic gain that arises when apolar surfaces are forced to interact, liberating water molecules in the bulk. Conversely, hydrogen bonding, ion pairing, and metal-ligand coordination are usually enthalpically driven. While it is relatively easy to select combinations of functional groups that are able to strongly interact by means of weak noncovalent intermolecular forces, it is not easy to predict for a given molecule how it will aggregate and which final supramolecular aggregate will form once it is placed in water. This explains why the number of well-defined aggregates and capsules assembled via the hydrophobic effect is largely smaller compared to hydrogen-bonded capsules, even though mastering of the hydrophobic effect for molecular recognition and supramolecular chemistry in general have great potential applications for a series of disciplines related to pharmaceutical chemistry and drug delivery.

\subsection{Dimeric capsule}

\subsubsection{Resorcinarene cavitand}

One of the most studied hydrophobically assembled dimeric capsule has been developed by Gibb and collaborators and is based on a deep partially water-soluble cavitand bearing eight carboxylic moieties and comprising a resorcin[4]arene scaffold with a second belt consisting of four aromatic rings to rigidify the structure. A third row of four aromatic residues is present to provide a hydrophobic rim that is involved in the dimerization process. ${ }^{118}$ Such octa-acid cavitand is soluble at millimolar concentration in $\mathrm{pH} 8.9$ buffered solutions in water and in the presence of suitable apolar guests of opportune size and shape undergo dimerization forming a hydrophobic cavity with volume of about $500-800 \AA^{3}$ that, depending on the size of the guest, can accommodate one or two guest molecules (Figure 23). ${ }^{119}$ It is the guest that, on the basis of its size and shape, acts as a template steering the formation of the assembly toward
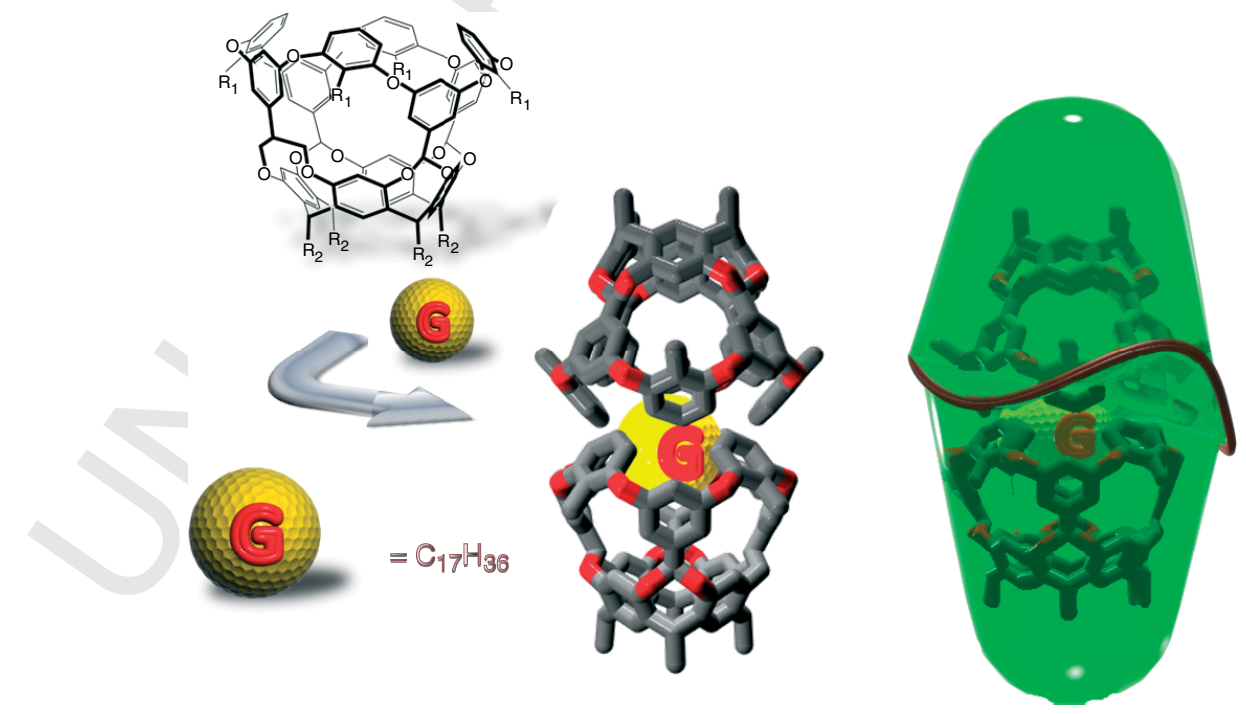

Figure 23 Hydrophobically driven dimerization of water-soluble octa-acid cavitand encapsulating apolar templating guests. 
dimeric capsules and decides the overall stoichiometry of the hydrophobic assembly.

Guest exchange is a rather slow process that takes place on the order of $0.1-1 \mathrm{~s}^{-1}$ and is governed by the assembly and disassembly process. There are many suitable guests, ranging from steroids, hydrocarbons from pentane through octadecane displaying high binding affinity $>10^{8} \mathrm{M}^{-1}$ and PCs between 48 and $73 \%$, but also smaller molecules such as gases like propane and butane with very high affinity for the latter, enabling the separation of mixtures of the two, simply equilibrating the gas with an aqueous solution of the hydrophobic cavitand. ${ }^{120}$

If the guest bound via the hydrophobic effect is also the substrate for a photocatalytic reaction, the dimeric capsule, owing to its high kinetic stability and the extremely short time frame of this kind of reactions, acts as a nanometric reaction chamber that is able to impart unique product selectivities. The system has been largely investigated for a series of reactions; selected examples are reported in the following, while a more exhaustive description can be found in the literature. ${ }^{119,121}$

Dibenzyl ketones are suitable guests for this dimeric capsule and they undergo exclusive formation of decarboxylation products in the absence of the hosting dimer, while in the presence of the capsule unusual massive formation of rearrangement products derived by recombination of radical residues and minor amounts of decarbonylation products is observed (Figure 24a). ${ }^{122}$ Moreover, as far as the decarbonylation products are concerned, photolysis within the capsule provided only mixed products and no homodimers, confirming that the recombination of radicals occurs in the capsule faster than in the in-out exchange.

Photocatalytic reactions of $\alpha$-alkyl dibenzoyl ketones were investigated along with the effect of the alkyl chain length that dictates the particular arrangement of the guest within the capsule and the subsequent product distribution as depicted in Figure 24(b), while in the absence of the capsule all the substrate led to the same pattern of products.

(a)
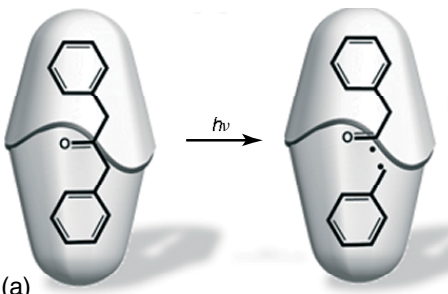

Benzyl radical $\stackrel{\text { rotation }}{\longrightarrow}$
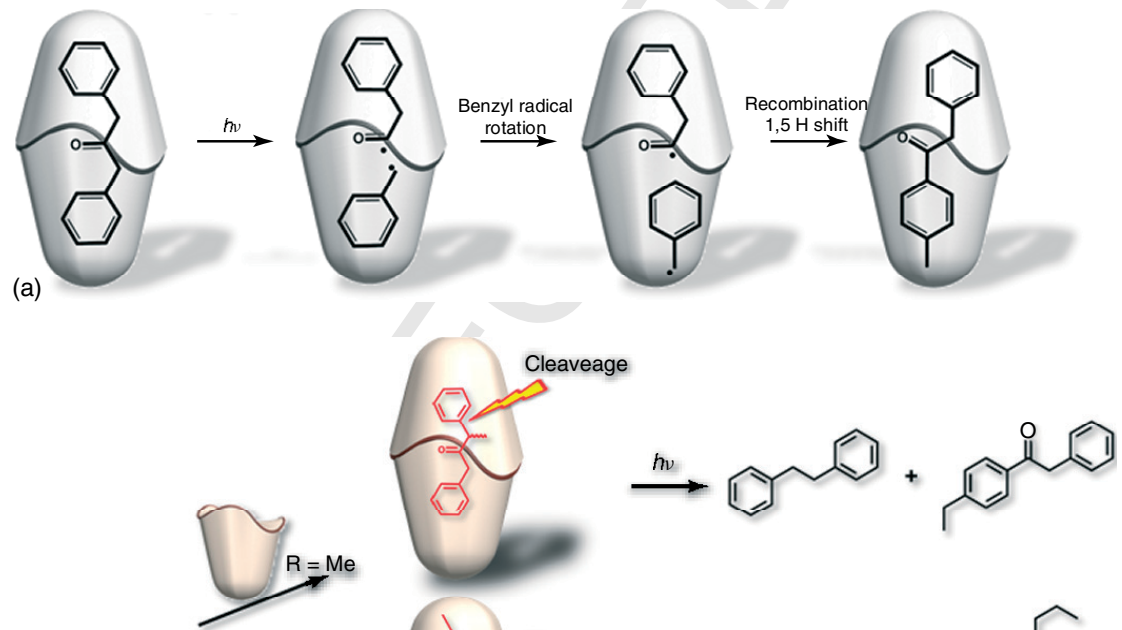

(b)

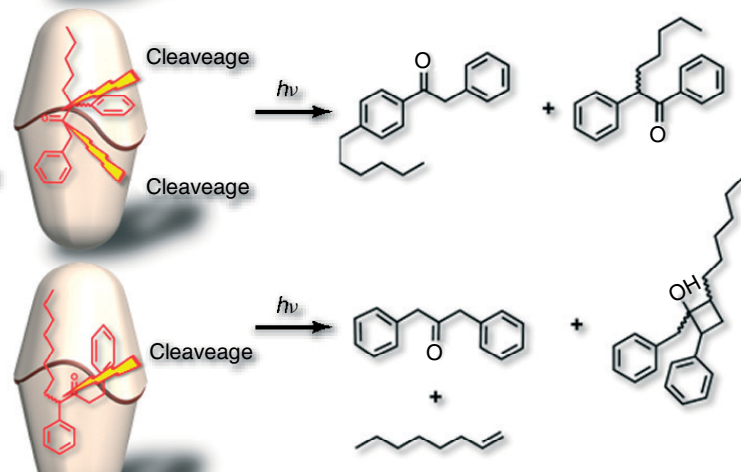

Figure 24 (a) Hydrophobic dimeric capsule leads to selective formation of 1-(4-methylphenyl)-2-phenylethanone with no decarbonylation side products in the photolysis of the encapsulated dibenzoyl ketone. (b) Photocatalytic cleavage of the encapsulated $\alpha$-alkyl dibenzoyl ketones undergo with different product distributions as a consequence of the length of the alkyl chain and the relative positioning within the capsule. 

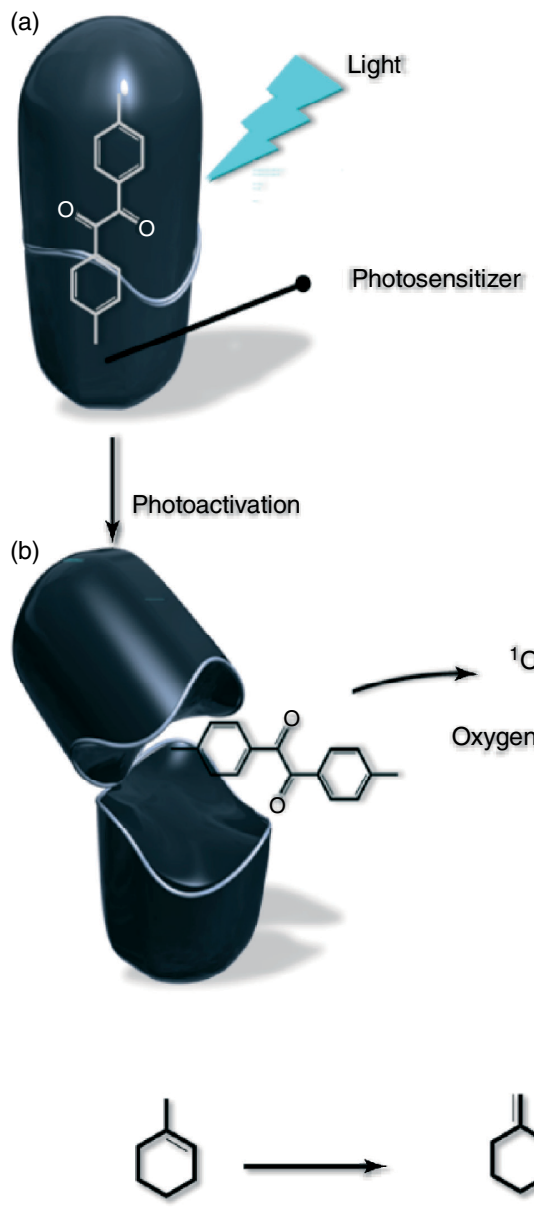

$\mathrm{RB} / \mathrm{CH}_{3} \mathrm{CN}$

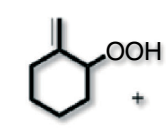

44
10 (d)

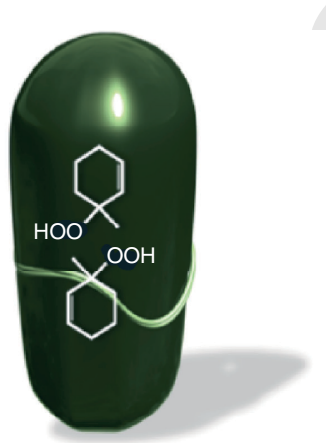

(c)

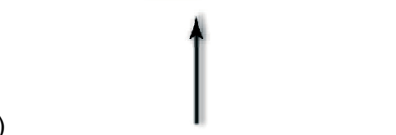

${ }^{1} \mathrm{O}_{2}$

Oxygen transfer
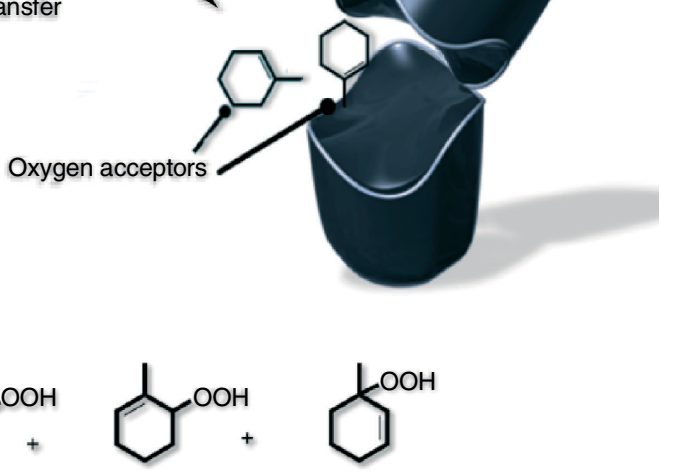

36

90

Figure 25 An encapsulated sensitizer is excited with light and it releases the energy to molecular oxygen that oxidizes a second hydrophobic guest selectively to the corresponding hydroperoxide.

For the encapsulated guests, with the methyl-substituted substrate, the aryl residues were placed on the tapered end of the capsule, while with the longer $n$-pentyl residue the alkyl group was placed toward the end of the cavity. Even longer $n$-octyl residues led to an even different substrate positioning with consequent different product distribution. $^{123}$

It was also possible to set up a photocatalytic reaction where singlet oxygen oxidation of cyclic alkenes was controlled by the capsule via interaction with a sensitizer like water-soluble Rose Bengal (RB) or insoluble dimethylbenzil (DMB) as sensitizers. ${ }^{124}$ All the alkenes provided quaternary $2: 2$ capsular self-assembled structures with the oxidation product distribution related to the position assumed in the cavity. The same reaction in acetonitrile without host led to a complex mixture of hydroperoxides (Figure 25).

\subsection{Hexameric and tetrameric capsules}

\subsubsection{Hexagram-shaped amphiphile}

The hydrophobic effect is very difficult to predict; in fact, even though it is rather simple to prepare amphiphilic molecules, it is highly difficult to predict the formation of discrete assemblies and often serendipity takes part in the game. One example is reported in Figure 26 where 


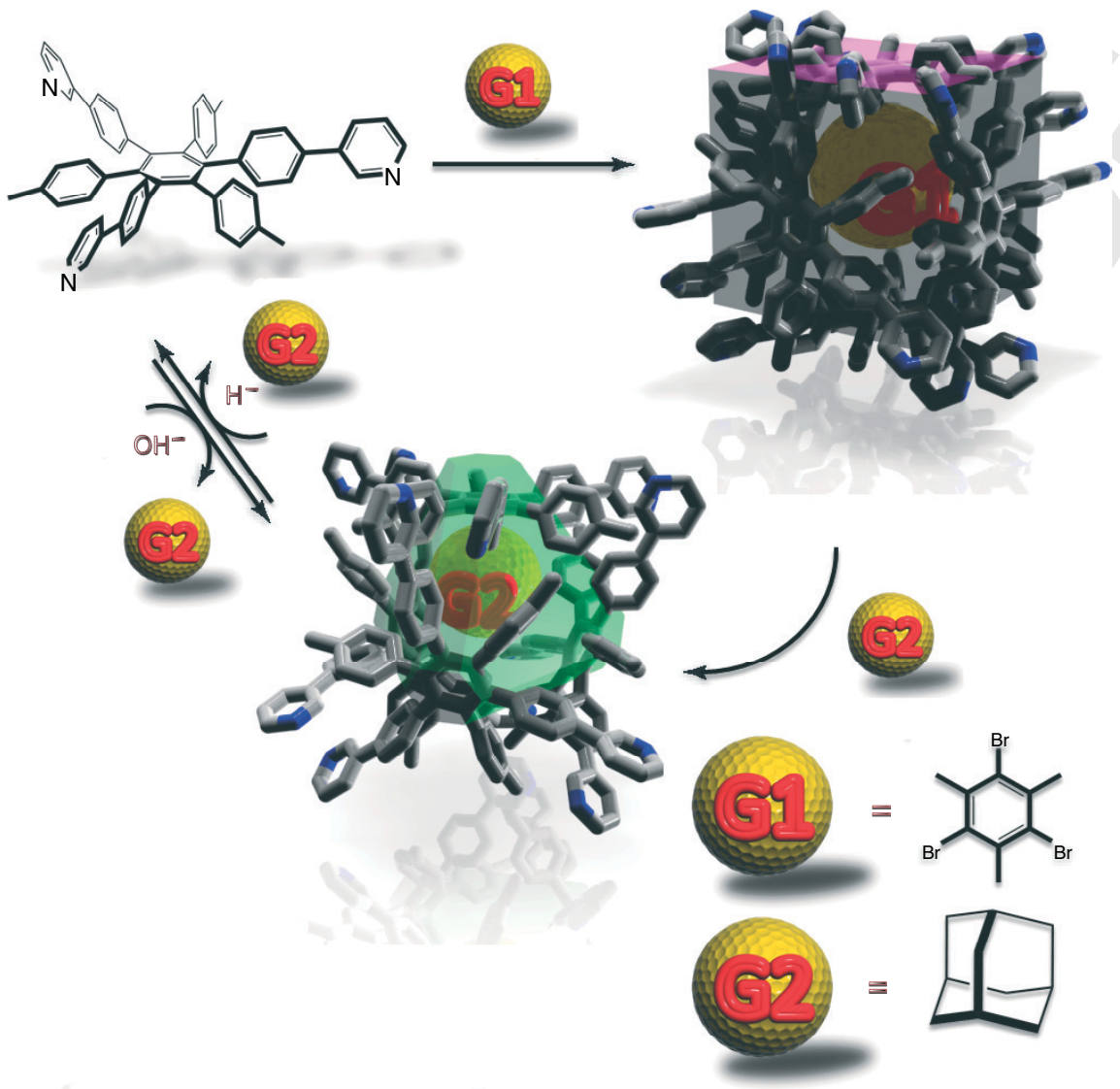

Figure 26 Amphiphilic hexagram-shaped molecules self-assemble via the hydrophobic effect, forming a hexameric box or a tetrameric tetrahedral-shaped capsule as a function of the templating hydrophobic guest.

the structure of the hexagram-shaped amphiphile molecule based on an aromatic apolar hexa-phenyl benzene core adorned with alternating pyridyl and methyl substituents is depicted. ${ }^{125}$ Such a molecule is singularly solvated in $\mathrm{CD}_{3} \mathrm{OD}$ providing signals on the NMR in accordance with its $C_{3}$ symmetry, but in the presence of increasing amounts of $\mathrm{D}_{2} \mathrm{O}$, new signals in the upfield region arise and, in $\mathrm{CD}_{3} \mathrm{OD}-\mathrm{D}_{2} \mathrm{O} 75: 25$, a hexameric boxlike aggregate is present that not only exists in solution but can also accommodate suitable guests like two molecules of tribromomesitylene that stack one another in the apolar core of the system (Figure 26 guest G1). It is worth noting that the presence of methyl residues on the monomer is crucial for the formation of the aggregate via the hydrophobic effect; in fact, without such residues the system does not provide the box aggregate but simply leads to multilayered structures. Van der Waals forces and $\mathrm{CH}-\pi$ interactions between such methyl groups and the adjacent aromatic rings help stabilize the discrete capsule structure of the hexameric aggregate to a great extent. As often described, the guest needs to complement the cavity provided by the hexameric supermolecule well, where hexamethylbenzene, mesitylene, and 1,3,5-tribromobenzene were suitable guests, while slightly larger hexabromobenzene or smaller 1,3,5-trichlorobenzene were not. Even more remarkable is the switch of the shape and nature of the self-assembled host induced by a more spherical guest such as adamantane, representing one of the most clear-cut examples of induced-fit assembly of the host to better complement the guest. Addition of 1.5 equivalents of adamantane with respect to the free hexameric assemble switches the aggregate toward a tetrahedral tetrameric new host that better complement the guest (Figure 26 guest G2). The structure of the new systems was unambiguously determined by X-ray analysis confirming its $C_{3}$ symmetry with the pyridine located between a cleft formed by other hexagramshaped molecules and the gust not close enough to the host thus supporting the encapsulation via hydrophobic 
effect rather that van del Waals interactions. The presence of the pyridine moieties allowed the reversible assembly and deassembly of the host-guest system by means of addition of 12 equivalents of $\mathrm{DCl}$ that protonate the nitrogen atoms, liberating adamantane and the monomer in solution as a tris-cationic species, while neutralization with $\mathrm{NaOD}$ returned the host-guest systems in its original tetrahedral form. ${ }^{126}$ The thermodynamic properties of the aggregation process of the amphiphilic monomer described above has been investigated in detail, and it was observed that aggregation does not occur under the classical entropic hydrophobic effect where the driving force is the release of water molecules that are more free to move, but via a nonclassical enthalpic hydrophobic effect that is present when the systems have extended aromatic $\pi$ surfaces that aggregate with enthalpically favorable $\pi-\pi$ contacts.

\section{CONCLUSIONS}

Organic self-assembled capsules have become increasingly sophisticated nanometric self-assembled devices over the years that are able to display unique control levels on a single or a few encapsulated molecules. The latter experience restricted rotation, tumbling, single molecule-to-molecule orientation, influenced chemical equilibria, and reactivity: all properties that cannot be controlled at the same level of sophistication for molecules in the bulk. Owing to the development of synthons like certain hydrogen-bonding moieties or particular scaffolds, it is possible to design and create new and more sophisticated capsules.

One of the most common comparisons involves supramolecular capsules and enzymes because they share many behaviors, properties, and common features. Encapsulation phenomena reveal new concepts that clearly show interaction between substrates and enzymes in the formation of active complexes that subsequently lead to catalytic reactions and product formation. Encapsulation criteria, and the relative driving forces involved, teach about substrate selectivity typical of enzymatic reactions, therefore the future development of new self-assembled capsules and the study of their encapsulation phenomena represents a test bench to master molecular recognition. Supramolecular capsules have already acted as supramolecular catalysts that are able to accelerate chemical transformation and to steer product selectivity, but turnover ability is still the critical point that requires improvements.

Another natural application of self-assembled capsules is the storage of particular chemicals, gases, for instance, or the delivery of drugs under certain conditions. These properties need increasing effort to be practicable but this is mainly dictated by the will.
It is therefore likely that the study of supramolecular encapsulation phenomena will increase in the near future boosted by the creativity and driven by the experience of talented scientists toward new results that will allow to bridge the gap between man-made and nature-made systems.

\section{ACKNOWLEDGMENTS}

Prof. J. Rebek Jr. is gratefully acknowledged for reviewing the manuscript.

\section{FURTHER READING}

Reviews and book chapters on organic self-assembled capsules. ${ }^{127-129}$

Reviews on supramolecular catalysis involving selfassembling organic capsules. ${ }^{121,130-132}$

\section{REFERENCES}

1. J. Badjić, A. Nelson, S. J. Cantrill, et al., Acc. Chem. Res., 2005, 38, 723 .

2. M. D. Pluth and K. N. Raymond, Chem. Soc. Rev., 2007, 36, $161-171$.

3. L. C. Palmer and J. Rebek Jr, Org. Biomol. Chem., 2004, 2, 3051 .

4. P. W. Atkins, Physical Chemistry, 4th edn, Oxford University Press, Oxford, 1993.

5. A. Pastor and E. Martínez-Viviente, Coord. Chem. Rev., 2008, 252, 2314.

6. S. Mecozzi and J. Rebek Jr, Chem. Eur. J., 1998, 4, 1016.

7. J. Rebek Jr., Angew. Chem. Int. Ed., 2005, 44, 2068.

8. J. Rebek Jr, Acc. Chem. Res., 2009, 42, 1660.

9. A. Scarso and J. Rebek Jr, Top. Curr. Chem., 2006, 265, 1.

10. B. C. Gibb, J. Supramol. Chem., 2002, 2, 123.

11. M. A. Mateos-Timoneda, M. Crego-Calama, and D. N. Reinhoudt, Chem. Soc. Rev., 2004, 33, 363.

12. J. Rebek Jr, Chem. Commun., 2007, 2777.

13. N. Branda, R. Wyler, and J. Rebek Jr, Science, 1994, 263, 1267.

14. C. Valdés, L. M. Toledo, U. Spitz, and J. Rebej Jr, Chem. Eur. J., 1996, 2, 989.

15. X. Garcías and J. Rebek Jr, Angew. Chem. Int. Ed., 1996 , 35, 1225.

16. T. Szabo, G. Hilmersson, and J. Rebek Jr, J. Am. Chem. Soc., 1998, 120, 6193.

17. N. Branza, R. M. Grotzfeld, C. Valdés, and J. Rebek Jr, J. Am. Chem. Soc., 1995, 117, 85. 
18. C. Valdés, U. P. Spitz, L. M. Toledo, et al., J. Am. Chem. Soc., 1995, 117, 12733.

19. A. Scarso, L. Pellizzaro, O. De Lucchi, et al., Angew. Chem. Int. Ed., 2007, 46, 4972.

20. M. Alajarín, R.-A. Orenes, J. W. Steed, and A. Pastor, Chem. Commun., 2010, 46, 1394.

21. M. Alajarín, A. Pastor, R.-A. Orenes, et al., Chem. Eur. J., 2004, 10, 1383.

22. M. Alajarín, A. Pastor, R.-A. Orenes, and J. W. Steed, J. Org. Chem., 2002, 67, 7091.

23. M. Alajarín, A. Pastor, R.-A. Orenes, et al., Chem. Eur. J., 2007, 13, 1559.

24. M. Alajarín, A. Pastor, R.-A. Orenes, et al., Chem. Commun., 2008, 3992.

25. J. Rebek Jr, Chem. Commun., 2000, 637.

26. V. Rudzevich, Y. Rudzevich, and V. Böhmer, Synlett, 2009, 12, 1887-1904.

27. K. D. Shimizu and J. Rebek Jr, Proc. Natl. Acad. Sci. U.S.A., 1995, 92, 12403.

28. A. M. Rincón, P. Prados, and J. de Mendoza, J. Am. Chem. Soc., 2001, 123, 3493.

29. R. K. Castellano, B. Hyean Kim, and J. Rebek Jr, J. Am. Chem. Soc., 1997, 119, 12671.

30. I. Thondorf, Y. Rudzevich, V. Rudzevich, and V. Böhmer, Org. Biomol. Chem., 2007, 5, 2775.

31. R. K. Castellano, C. Nuckolls, and J. Rebek Jr, J. Am. Chem. Soc., 1999, 121, 11156.

32. A. Pop, M. O. Vysotsky, M. Saadioui, and Volker Böhmer, Chem. Commun., 2003, 1124.

33. B. Kuberski and A. Szumna, Chem. Commun., 2009, 1959.

34. A. Szumna, Chem. Commun., 2009, 4191.

35. A. Szumna, Chem. Eur. J., 2009, 15, 12381.

36. R. E. Brewster and B. Shuker, J. Am. Chem. Soc., 2002, 124, 7902.

37. P. Ballester and G. Gil-Ramírez, Proc. Natl. Acad. Sci. U.S.A., 2009, 106, 10455.

38. G. Gil-Ramírez, M. Chas, and P. Ballester, J. Am. Chem. Soc., 2010, 132, 2520.

39. M. Chas, G. Gil-Ramírez, E. C. Escudero-Adán, et al., Org. Lett., 2010, 12, 1740.

40. J. Kang and J. Rebek Jr, Nature, 1996, 382, 239.

41. Y. Tokunaga and J. Rebek Jr, J. Am. Chem. Soc., 1998, 120, 66.

42. A. Scarso and G. Borsato, Optically active supramolecules, in ed. D. B. Amabilino, Chirality at the Nanoscale, WileyVCH, Weinheim, 2009, 29.

43. J. M. Rivera, T. Martín, and J. Renek Jr, Science, 1998, 279, 1021.

44. J. M. Rivera, T. Martín, and J. Rebek Jr, J. Am. Chem. Soc., 2001, 123, 5213.

45. J. M. Rivera, S. L. Craig, T. Martín, and J. Rebek Jr, Angew. Chem. Int. Ed., 2000, 39, 2130.

46. J. Kang and J. Rebek Jr, Nature, 1997, 385, 50.
47. J. Kang, J. Santamaria, G. Hilmersson, and J. Rebek Jr, J. Am. Chem. Soc., 1998, 120, 7389.

48. S. K. Körner, F. C. Tucci, D. M. Rudkevich, et al., Chem. Eur. J., 2000, 6, 187.

49. M. Yamanaka and J. Rebek Jr, Chem. Commun., 2004, 1690.

50. T. Amaya and J. Rebek Jr, Chem. Commun., 2004, 1802.

51. A. Shivanyuk and J. Rebek Jr, J. Am. Chem. Soc., 2002, 124, 12074.

52. A. Scarso and J. Rebek Jr, J. Am. Chem. Soc., 2004, 126, 8956.

53. A. Scarso, H. Onagi, and J. Rebek Jr, J. Am. Chem. Soc., 2004, 126, 12728

54. A. Shivanyuk, A. Scarso, and J. Rebek Jr, Chem. Commun., 2003, 1230.

55. T. Amaya and J. Rebek Jr, J. Am. Chem. Soc., 2004, 126, 14149.

56. A. Scarso, L. Trembleau, and J. Rebek Jr, J. Am. Chem. Soc., 2004, 126, 13512.

57. A. Scarso, L. Trembleau, and J. Rebek Jr, Angew. Chem. Int. Ed., 2003, 42, 5499.

58. T. Heinz, D. M. Rudkevich, and J. Rebek Jr, Angew. Chem. Int. Ed., 1999, 38, 1136.

59. L. C. Palmer, Y.-L. Zhao, K. N. Houk, and J. Rebek Jr, Chem. Commun., 2005, 3667.

60. A. Scarso, A. Shivanyuk, O. Hayashida, and J. Rebek Jr, J. Am. Chem. Soc., 2003, 125, 6239.

61. M. P. Schramm, P. Restorp, F. Zelder, and J. Rebek Jr J. Am. Chem. Soc., 2008, 130, 2450.

62. T. Amaya and J. Rebek Jr, J. Am. Chem. Soc., 2004, 126, 6216.

63. J. Chen and J. Rebek Jr, Org. Lett., 2002, 4, 327.

64. J.-L. Hou, D. Ajami, and J. Rebek Jr, J. Am. Chem. Soc., 2008, 130, 7810.

65. T. Martín, U. Obst, and J. Rebek Jr, Science, 1998, 281, 1842 .

66. D. W. Johnson, F. Hof, P. M. Iovine, et al., Angew. Chem. Int. Ed., 2002, 41, 3793.

67. F. Hof, C. Nuckolls, S. L. Craig, et al., J. Am. Chem. Soc., 2000, 122, 10991

68. C. Nuckolls, F. Hof, T. Martin, and J. Rebek Jr, J. Am. Chem. Soc., 1999, 121, 10281.

69. L. R. MacGillivray and J. L. Atwood, Nature, 1997, 389, 469.

70. E. S. Barrett, T. J. Dale, and J. Rebek Jr, J. Am. Chem. Soc., 2008, 130, 2344.

71. L. Avram and Y. Cohen, Org. Lett., 2002, 4, 4365.

72. A. Shivanyuk and J. Rebek, Jr, Proc. Natl. Acad. Sci. U.S.A., 2001, 98, 7662.

73. M. Yamanaka, A. Shivanyuk, and J. Rebek Jr, J. Am. Chem. Soc., 2004, 126, 2939.

74. A. Shivanyuk and J. Rebek Jr, Chem. Commun., 2001, 2424. 
75. I. E. Philip and A. E. Kaifer, J. Am. Chem. Soc., 2002, 124, 12678.

76. A. Shivanyuk, J. Am. Chem. Soc., 2007, 129, 14196.

77. L. C. Palmer, A. Shivanyuk, M. Yamanaka, and J. Rebek Jr, Chem. Commun., 2005, 857.

78. T. Evan-Salem, I. Baruch, L. Avram, et al., Proc. Natl. Acad. Sci. U.S.A., 2006, 103, 12296.

79. L. Avram and Y. Cohen, J. Am. Chem. Soc., 2004, 126, 11556.

80. J. L. Atwood, L. J. Barbour, and A. Jerga, Chem. Commun., 2001, 2376.

81. L. Avram and Y. Cohen, Org. Lett., 2006, 8, 219.

82. L. Avram and Y. Cohen, J. Am. Chem. Soc., 2003, 125, 16180.

83. L. Palmer and J. Rebek Jr, Org. Lett., 2005, 7, 787.

84. S. J. Dalgarno, S. A. Tucker, D. B. Bassil, and J. L. Atwood, Science, 2005, 309, 2037.

85. K. Kobayashi, K. Ishii, S. Sakamoto, et al., J. Am. Chem. Soc., 2003, 125, 10615.

86. K. Kobayashi, K. Ishii, and M. Yamanaka, Chem. Eur. J., 2005, 11, 4725.

87. J. Nakazawa, M. Mizuki, Y. Shimazaki, et al., Org. Lett., 2006, 8, 4275.

88. K. Kobayashi, R. Kitagawa, Y. Yamada, et al., J. Org. Chem., 2007, 72, 3242.

89. H. Kitagawa, M. Kawahata, R. Kitagawa, et al., Tetrahedron, 2009, 65, 7234.

90. H. Kitagawa, Y. Kobori, M. Yamanaka, et al., Proc. Natl. Acad. Sci. U.S.A., 2009, 106, 10444.

91. D. Ajami, M. P. Schramm, A. Volonteiro, and J. Rebek Jr, Angew. Chem. Int. Ed., 2007, 46, 242.

92. D. Ajami, T. J. Dale, E. Barrett, and J. Rebek Jr, Proc. Natl. Acad. Sci. U.S.A., 2009, 106, 10430.

93. K. Kobayashi, T. Shirasaka, K. Yamaguchi, et al., Chem. Commun., 2000, 41.

94. M. Yamanaka, K. Ishii, Y. Yamada, and K. Kobayashi, J. Org. Chem., 2006, 71, 8800.

95. D Ajami and J. Rebek Jr, J. Am. Chem. Soc., 2006, 128, 5314.

96. D. Ajami and J. Rebek Jr, Nature Chem., 2009, 1, 87.

97. D. Ajami and J. Rebek Jr, J. Am. Chem. Soc., 2006, 128, 15038 .

98. D. Ajami and J. Rebek Jr, Angew. Chem. Int. Ed., 2007, 46, 9283 .

99. D. Ajami and J. rebek Jr, Angew. Chem. Int. Ed., 2008, 47, 6059.

100. D. Ajami and J. Rebek Jr, J. Org. Chem., 2009, 74, 6584.

101. D. Ajami and J. Rebek Jr, Proc. Natl. Acad. Sci. U.S.A., 2007, 104, 16000.

102. L. J. Prins, P. Timmerman, and D. N. Reinhoudt, J. Am. Chem. Soc., 2001, 123, 10153.

103. J. M. C. A. Kerckhoffs, M. G. J. ten Cate, M. A. MateosTimoneda, et al., J. Am. Chem. Soc., 2005, 127, 12697.
104. J. M. C. A. Kerckhoffs, F. W. B. van Leeuwen, A. L. Spek, et al., Angew. Chem. Int. Ed., 2003, 42, 5717.

105. M. G. J. ten Cate, D. N. Reinhoudt, and M. Crego-Calama, J. Org. Chem., 2005, 70, 8443.

106. M. A. Mateos-Timoneda, J. M. C. A. Kerckhoffs, M. Crego-Calama, and D. N. Reinhoudt, Angew. Chem. Int. Ed., 2005, 44, 3248.

107. R. Fiammengo, P Timmerman, F. de Jong, and D. N. Reinhoudt, Chem. Commun., 2000, 2313.

108. R. Fiammengo, K. Wojciechowski, M. Crego-Calama, et al., Org. Lett., 2003, 5, 3367.

109. R. Zadmard, T. Schrader, T. Grawe, and A. Kraft, Org. Lett., 2002, 4, 1687.

110. R. Zadmard, M. Junkers, T. Schrader, et al., J. Org. Chem., 2003, 68, 6511 .

111. F. Corbellini, F. W. B. van Leeuwen, H. Beijleveld, et al., New J. Chem., 2005, 29, 243.

112. J. S. Sasine, R. E. Brewster, K. L. Caran, et al., Org. Lett., 2006, 8, 2913.

113. F. Corbellini, R. Fiammengo, P. Timmerman, et al., J. Am. Chem. Soc., 2002, 124, 6569.

114. F. Corbellini, L. Di Costanzo, M. Crego-Calama, et al., J. Am. Chem. Soc., 2003, 125, 9946.

115. F. Corbellini, R. M. A. Knegtel, P. D. J. Grootenhuis, et al., Chem. Eur. J., 2005, 11, 298.

116. H.-J. Kim, S. Sakamoto, K. Yamaguchi, and J.-I. Hong, Org. Lett., 2003, 5, 1051

117. G. V. Oshovsky, D. N. Reinhoudt, and W. Verboom, J. Am. Chem,. Soc., 2006, 128, 5270.

118. C. L. D. Gibb and B. C. Gibb, J. Am. Chem. Soc., 2004, 126, 11408

119. S. Liu and B. C. Gibb, Chem. Commun., 2008, 32, 3709.

120. C. L. D. Gibb and B. C. Gibb, J. Am. Chem. Soc., 2006, 128, 16498.

121. A. Scarso and G. Borsato, Capsules and cavitands: artificial catalysts of nanometric dimension, in E. Groppo, S. Bordiga, V. Zecchina, Selective Nanocatalysis and Nanoscience, Concepts for Heterogeneous and Homogeneous Catalysis, Wiley-VCH, Weinheim,• 2010, in press.

122. L. S. Kaanumalle, C. L. D. Gibb, B. C. Gibb, and V. Ramamurthy, J. Am. Chem. Soc., 2004, 126, 14366.

123. C. L. D. Gibb, A. K. Sundaresan, V. Ramamurthy, and B. C. Gibb, J. Am. Chem. Soc., 2008, 130, 4069.

124. A. Natarajan, L. S. Kaanumalle, et al., J. Am. Chem. Soc., 2007, 129, 4132.

125. S Hiraoka, K. Harano, M. Shiro, and M. Shionoya, J. Am. Chem. Soc., 2008, 130, 14368.

126. S. Hiraoka, K. Harano, T. Nakamura, et al., Angew. Chem. Int. Ed., 2009, 48, 7006.

127. M. M. Conn and J. Rebek Jr, Chem. Rev., 1997, 97, 1647.

128. J. Rebek Jr. Acc. Chem. Res., 1999, 32, 278.

129. P. Ballester and J. de Mendoza, Supramolecular macrocycle synthesis by H-bonding assembly, in F. Diederich, 
P. J. Stang, and R. R. Tykwiski, Modern Supramolecular Chemistry, Wiley-VCH, Weinheim, 2008.

130. P. W. N. M. Van Leeuwen, Supramolecular Catalysis, Wiley-VCH, Weinheim, 2008.
131. T. S. Koblenz, J. Wassenaar, and J. N. H. Reek, Chem. Soc. Rev., 2007, 37, 247.

132. D. M. Vriezema, M. Comellas Aragonés, J. A. A. W. Elemans, et al., Chem. Rev., 2005, 105, 1445. 
Please note that the abstract and keywords will not be included in the printed book, but are required for the online presentation of this book which will be published on Wiley's online platform. If the abstract and keywords are not present below, please take this opportunity to add them now.

The abstract should be a short paragraph up to 200 words in length and there should be between five and ten keywords.

ABSTRACT: The present contribution is aimed at illustrating to the reader the state of the art on the construction of artificial, finite supramolecular assemblies characterized in solution by capsular shape held together by means of weak intermolecular noncovalent forces in solution, except metal-ligand coordination. These supramolecular assemblies provide cavities of correct size and shape where molecules of guests present in solution can be hosted if the available space is appropriately filled. Guests within the cavity are held for lifetimes ranging from milliseconds to hours, depending on the number and quality of weak intermolecular forces and the number of subunits that compose the capsule.

The in-out process of exchange of guests influences their behavior profoundly, enabling a series of applications spanning from molecular recognition of one up to several guests at a time with creation of new forms of isomerism derived by guest-guest interactions to supramolecular catalysis, which is the ultimate goal of such kind of assemblies.

A further intriguing aspect of self-assembled capsules is chirality that can be implemented in the subunits, that forms the capsule, or that can be the result of a particular arrangement of achiral units. In the latter case, the presence of strong attractive forces between the subunits enables to template the formation of enantiomerically enriched capsules with enantiopure guests based on achiral units via the chiral memory effect.

KEYWORDS: self-assembled capsules, hydrogen bonding, ion pairing, hydrophobic effect, encapsulation, packing coefficient, supramolecular chirality, supramolecular catalysis, orientational isomerism, social isomerism 


\section{QUERIES TO BE ANSWERED BY AUTHOR (SEE MARGINAL MARKS Q..)}

IMPORTANT NOTE: You may answer these queries by email. If you prefer, you may print out the PDF, and mark your corrections and answers directly on the proof at the relevant place. Do NOT mark your corrections on this query sheet. Please see the proofing instructions for information about how to return your corrections and query answers.

Q1. Please clarify if this article has since been published. If so, please provide the page range for reference 121. 